\title{
THESIS
}

\section{RETINAL GANGLION CELL DISTRIBUTION AND VISUAL ACUITY IN ALPACAS}

(Vicugna pacos)

\author{
Submitted by \\ Hsiao-Hui Wang \\ Department of Biomedical Sciences
}

In partial fulfillment of the requirements

For the degree of Master of Science

Colorado State University

Fort Collins, Colorado

Fall 2013

Master's Committee:

Advisor: James Madl

Co-advisor: Juliet Gionfriddo

Stacey Byers

Jozsef Vigh 


\section{ABSTRACT \\ RETINAL GANGLION CELL DISTRIBUTION AND VISUAL ACUITY IN ALPACAS}

(Vicugna pacos)

Vision reflects the visual demand for environmental adaptation of an animal and also implies the biological niche of a species. Retinal specializations, such as visual streaks, areae centrales and foveae, have been found in animals and the function of each retinal specialization is correlated to its visual needs. Harman et al analyzed retinal ganglion cell (RGC) topography of dromedary camels and found a vertical steak which is unusual for ungulates. A vertical steak had only been reported in two-toed sloths. Because alpacas are closely related to dromedary camels in the same taxonomic family, we hypothesized that alpacas have similar RGC topography as do dromedary camels rather than other grazing animals.

Five healthy alpaca eyes were included in the current study. Anti-Brn-3a antibody was used to label the RGCs and $25 \%$ of the retinal area was imaged to quantify the RGCs. Two other RGC staining techniques, Lucifer Yellow retrograde labeling and cresyl violet staining, were attempted but failed. A topographic retinal map of RGC densities was reconstructed based on the RGC counts. The axial length of each eye was measured and the measurement was used to calculate the visual acuity of alpacas.

The results of the Brn-3a labeling showed that alpacas have a horizontal streak across the retinal meridian superior to the optic disc with an upward extension at the temporal end of the streak. The highest RGC density area of the five retinas was in the temporal region. The maximal visual acuity, located in the temporal retina, ranged between 12.5 and 13.4 cycles per degree. 
Based on the results, we concluded that the RGC topography of alpacas was similar to that of other grazing animals, such as ox, sheep and horses, rather than that of dromedary camels. 


\section{ACKNOWLEDGEMENTS}

I would like to thank Dr. Juliet Gionfriddo for all her time and support to the project and my thesis. Her professional knowledge and valuable advice also helps me in my future career goal.

Also, I'm grateful to have Drs. Byers, Madl and Vigh to be my committee members. I would like to thank them for providing me the guidance and all the assistance.

I would like to thank Shannon Gallagher who has been very encouraging all the time and trained me on the concepts and techniques of immunohistochemistry.

I would like to thank Brad Charles and Todd Bass who have been providing all the technical support.

Last but not least, I would like to express my deepest appreciation to my parents who have been showing their care and providing the strongest support. 


\section{TABLE OF CONTENTS}

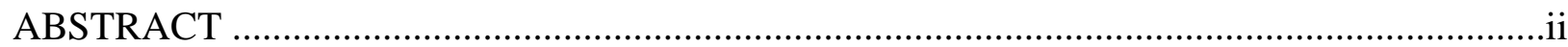

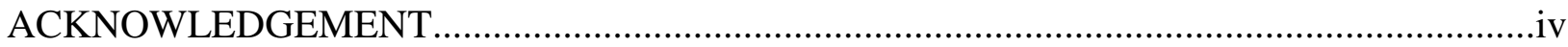

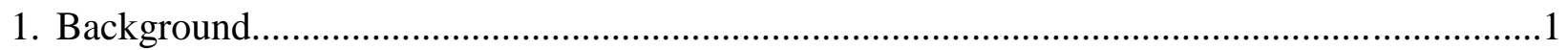

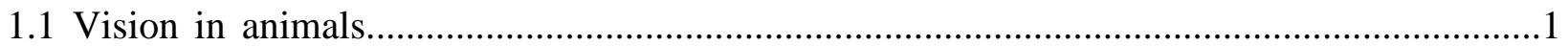

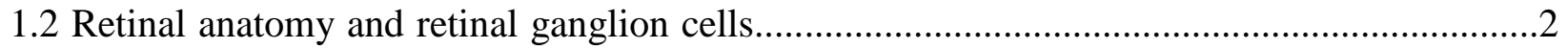

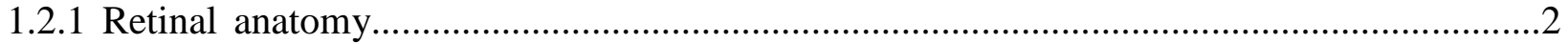

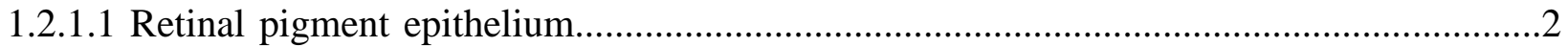

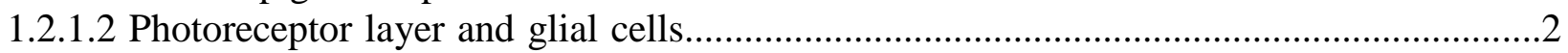

1.2.1.3 Outer nuclear layer and outer plexiform layer..............................................................

1.2.1.4 Inner nuclear layer and inner plexiform layer...........................................................

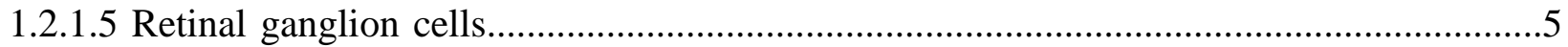

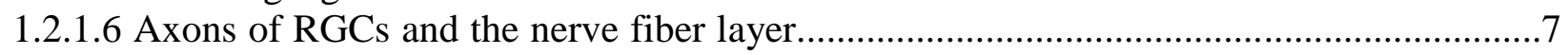

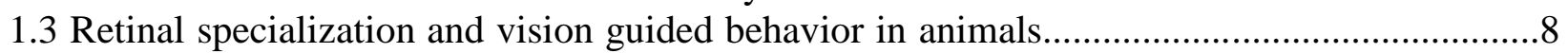

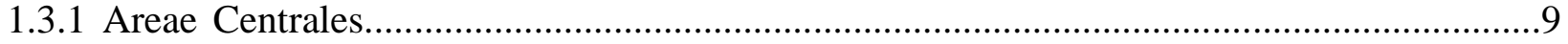

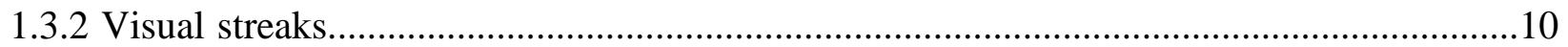

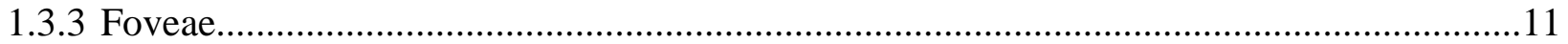

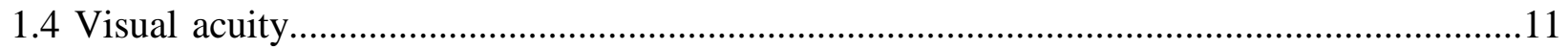

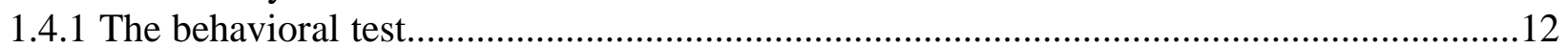

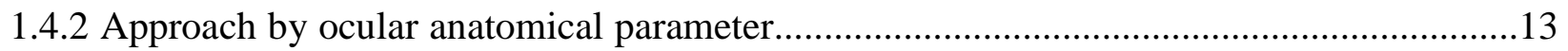

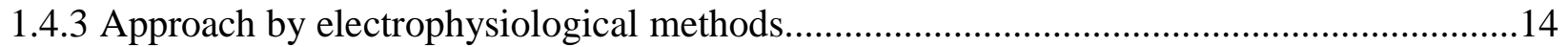

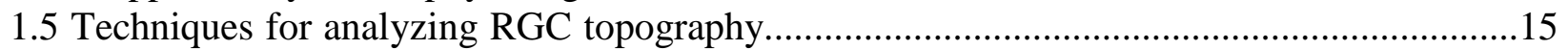

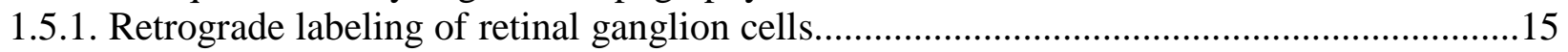

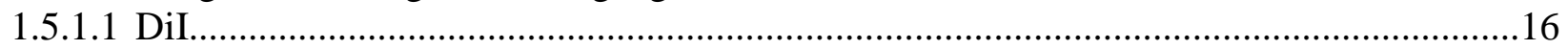

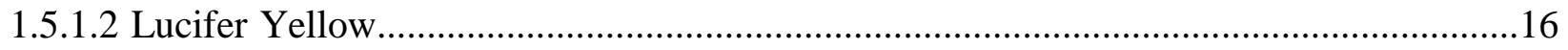

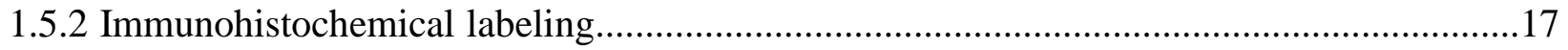

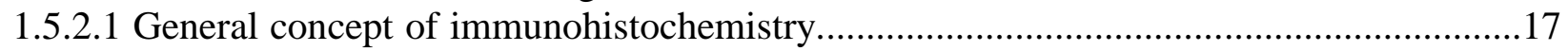

1.5.2.2 Retinal ganglion cell markers and the Brn-3 family..................................................18

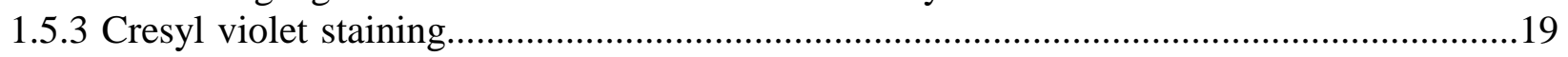

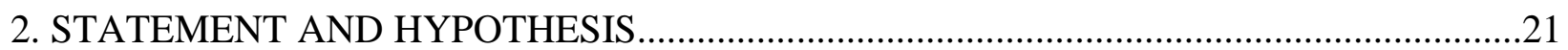

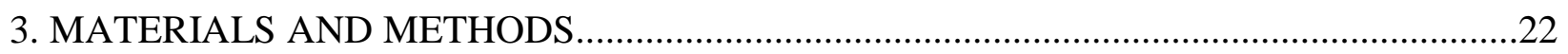

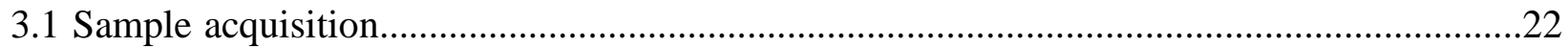

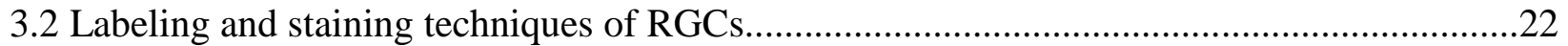

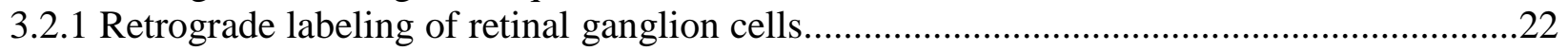

3.2.2 Tissue preparation for immunohistochemical study ........................................................23

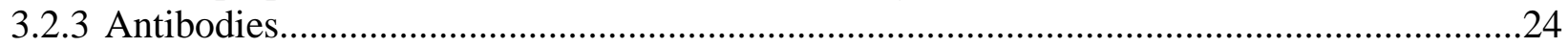

3.2.4 Study for Lucifer Yellow labeling and anti-Lucifer Yellow antibody labeling on cross-

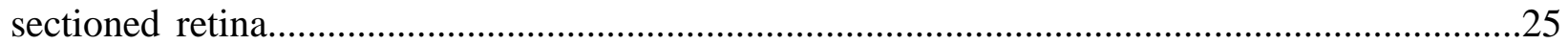


3.2.4.1 Evaluating retrograde labeling of Lucifer Yellow labeling on cross-sectioned retina......25 3.2.4.2 Evaluating retrograde labeling of Lucifer Yellow labeling with anti-Lucifer Yellow

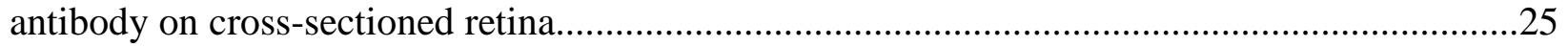

3.2.5 Immunohistochemical labeling of retinal ganglion cells with anti-Brn-3a antibody..........25

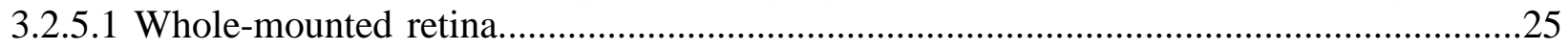

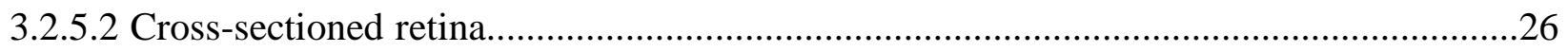

3.2.6 Cresyl violet staining of retinal ganglion cells........................................................26

3.3 Osmication of the myelin of the optic nerve fibers......................................................28

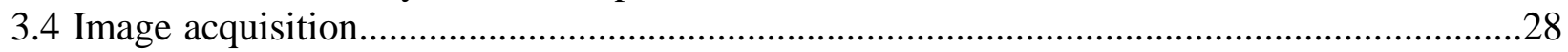

3.4.1 Study for Lucifer Yellow labeling and anti-Lucifer Yellow antibody labeling on cross-

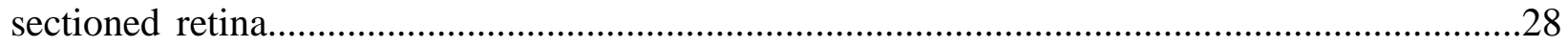

3.4.1.1 Evaluating retrograde labeling of Lucifer Yellow labeling on cross-sectioned retina......29 3.4.1.2 Evaluating retrograde labeling of Lucifer Yellow labeling with anti-Lucifer Yellow antibody on cross-sectioned retina...............................................................................29

3.4.2 Immunohistochemical labeling of retinal ganglion cells with anti-Brn-3a antibody..........29

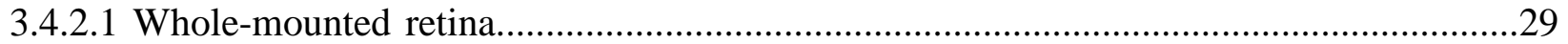

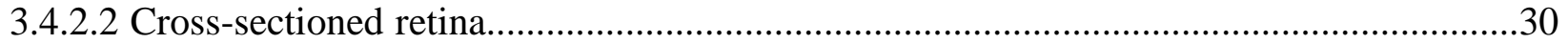

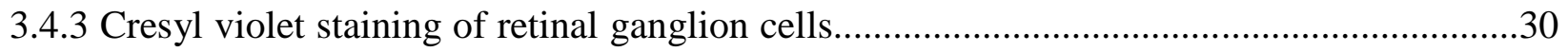

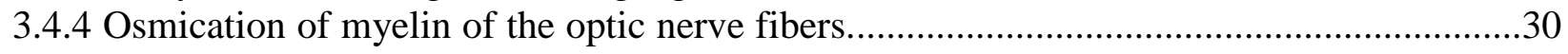

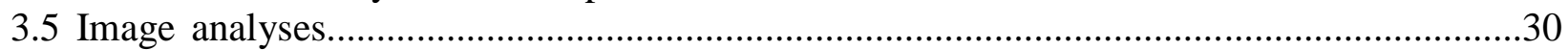

3.5.1 Study for Lucifer Yellow labeling and anti-Lucifer Yellow antibody labeling on cross-

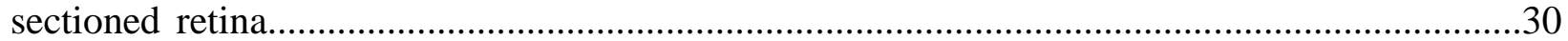

3.5.1.1 Evaluating retrograde labeling of Lucifer Yellow labeling on cross-sectioned retina......31

3.5.1.2 Evaluating retrograde labeling of Lucifer Yellow labeling with anti-Lucifer Yellow

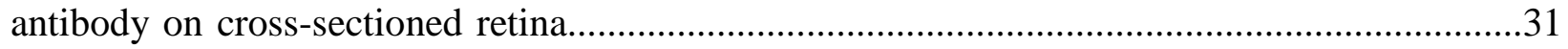

3.5.2 Immunohistochemical labeling of retinal ganglion cells with anti-Brn-3a antibody...........31

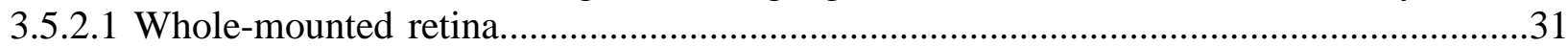

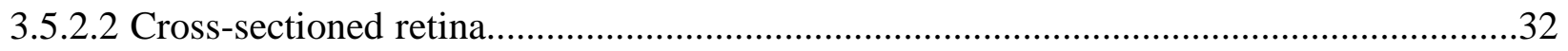

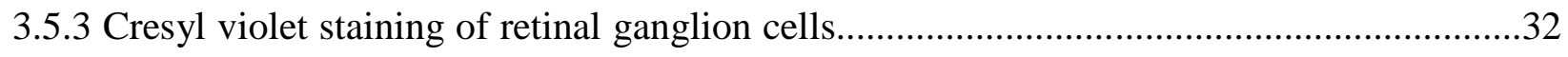

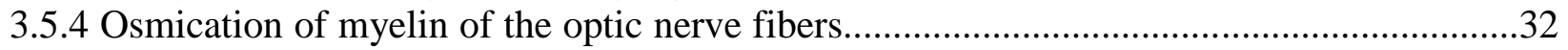

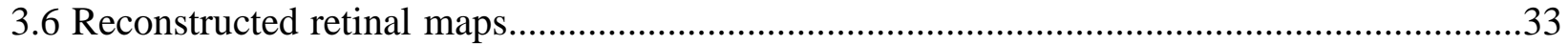

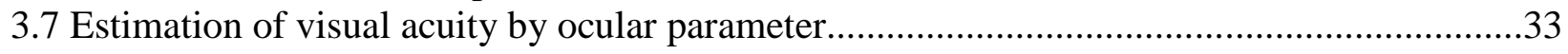

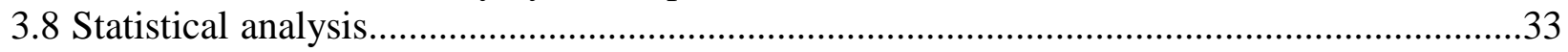

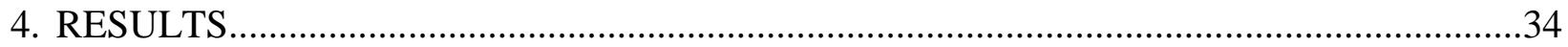

4.1 Labeling and staining techniques of retinal ganglion cells.............................................34

4.1.1 Study for Lucifer Yellow labeling and anti-Lucifer Yellow antibody labeling on cross-

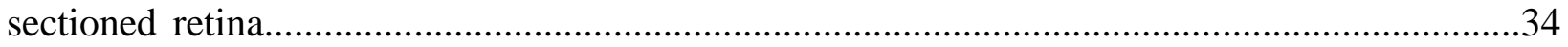

4.1.1.1 Evaluating retrograde labeling of Lucifer Yellow labeling on cross-sectioned retina......34 4.1.1.2 Evaluating retrograde labeling of Lucifer Yellow labeling with anti-Lucifer Yellow

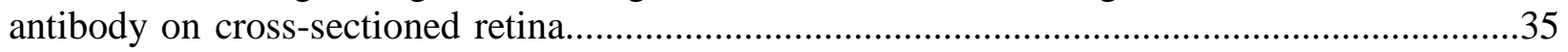

4.1.2 Immunohistochemical labeling of retinal ganglion cells with anti-Brn-3a antibody...........36

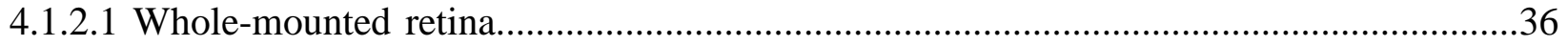

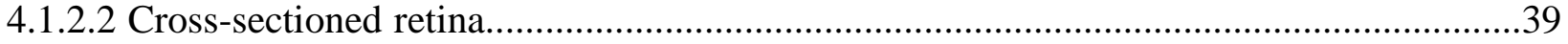

4.1.3 Cresyl violet staining of retinal ganglion cells......................................................40 
4.2 Osmication of myelin of the optic nerve fibers..........................................................

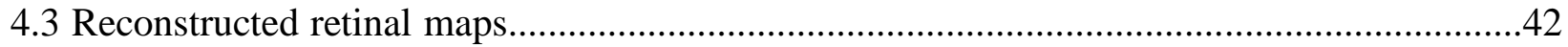

4.4 Estimation of visual acuity by ocular parameter........................................................43

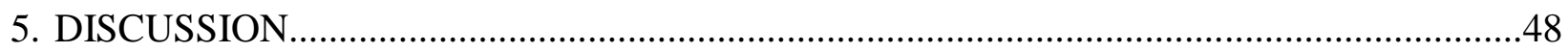

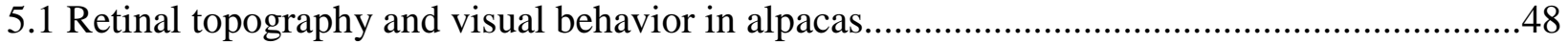

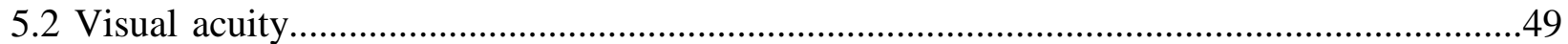

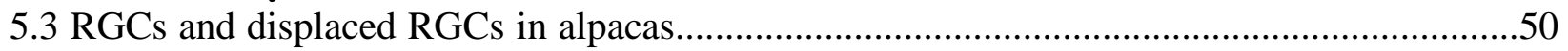

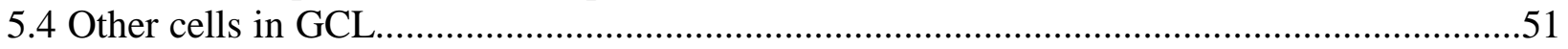

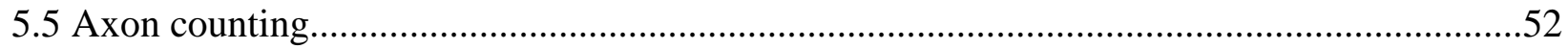

5.6 Unsuccessful attempts in Lucifer Yellow retrograde labeling ........................................53

5.7 Unsuccessful attempts to use Cresyl violet staining in RGC counting...............................54

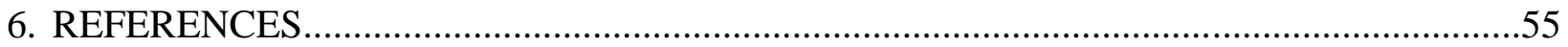




\section{BACKGROUND}

\subsection{Vision in animals}

Vision is of great importance for all animals, for it reflects not only the visual demand for environmental adaptation but also the ecological niche of a species. ${ }^{1}$ The visual pathway involves a series of sophisticated processes extending from the eyes to the visual cortex in the brain, and the pathway is highly variable among species. ${ }^{2}$

The visual pathway starts in the eye when the light goes through the optic axis. ${ }^{3}$ After arriving at the retina, the energy is either transformed into neuronal signals by photoreceptors or absorbed by the retinal pigmented epithelium. ${ }^{4}$ Then the signals are transmitted to interneurons including bipolar cells, amacrine cells and horizontal cells. ${ }^{4}$ After the signal modifying processes in the interneurons, the information is eventually delivered to the retinal ganglion cells (RGCs) and is further conducted to the retino-recipient zones in the brain via the optic nerve and optic tracts. $^{5}$

In most mammals, RGCs project their axons to several regions in the brain which are also known as retino-recipient zones. ${ }^{2}$ These areas include suprachiasmatic nuclei, the accessory optic nuclei, the pretectal nuclei, the ventral lateral geniculate nuclei (LGN), the dorsal LGN and the superior colliculi. ${ }^{2,6}$ The number and percentage of axon projection to each region are highly variable among animal species. ${ }^{2}$

Because of the significant variations in the habitat and behavior of animals, the distributions of the retinal neurons, mainly photoreceptors and RGCs, have been investigated in several species in order to reveal the relationship between the retinal topography and visionguided behavior. ${ }^{1}$ 
1.2 Retinal anatomy and retinal ganglion cells

\subsubsection{Retinal anatomy}

The retina is a very thin tissue at the posterior part of the eyes and is considered as part of the central nervous system. ${ }^{5}$ There are ten layers in the retina and the outermost layer is retinal pigment epithelium (RPE) located next to the neurosensory retina. ${ }^{7}$ The other 9 layers of the neurosensory retina include the photoreceptor layer, external limiting membrane, outer nuclear layer, outer plexiform layer, inner nuclear layer, inner plexiform layer, ganglion cell layer, nerve fiber layer and internal limiting membrane. ${ }^{7}$

\subsubsection{Retinal pigment epithelium}

The RPE is a layer of pigmented cells which forms the outermost retina. ${ }^{4}$ When the light reaches the retina, part of the light energy is absorbed by the outer segments of the photoreceptors and the rest is absorbed by RPE. ${ }^{4}$ In some animals with a tapetum, the RPE in the tapetal region has no pigment. ${ }^{4}$ Instead, the tapetum acts as a mirror to reflect the light back to the photoreceptors which is not absorbed in the first pass. ${ }^{4}$ This specific feature increases the light which is absorbed by the outer segments of the photoreceptors and helps with the scotopic vision. ${ }^{4}$ The RPE also recycles the bleached photopigments of the photoreceptors and maintains the metabolism of the retina. ${ }^{4}$

\subsubsection{Photoreceptor layer and glial cells}

The photoreceptor layer is composed of the inner and outer segments of the photoreceptors. ${ }^{4}$ This is the layer in which phototransduction begins. ${ }^{4}$ For example, when the light energy arrives, the chromophores in the photopigments of the rods separate from the opsin 
and then transform from 11-cis retinal aldehyde into all-trans retinal aldehyde. ${ }^{3,4}$ After a cascade of processes involving G proteins, the final result is the decrease in cGMP, the closure of the sodium channel in the outer segments and hyperpolarization of the photoreceptors., 3

The external limiting membrane is made up of the cell membrane of the rods, cones and Müller cells. ${ }^{4}$ Müller cells extend from the external limiting membrane to the internal limiting membrane and serve as the structural skeleton of the retina. ${ }^{4}$ Müller cells also synthesize and store glycogen which protects the retina from the systemic blood glucose fluctuation. ${ }^{3}$ Additionally, Müller cells regulate the ionic balance in the retina by uptaking the potassium ion secreted by photoreceptors. ${ }^{3}$ Müller cells also uptake and metabolize the neutrotransmitters, including $\gamma$-aminobutyric acid (GABA), dopamine and glutamate. ${ }^{3}$ Besides Müller cells, there are other types of neuroglial cells in the retina, astrocytes and oligodendrocytes. ${ }^{4}$ The nuclei of neuroglial cells are mainly in the inner nuclear layer (INL). ${ }^{4}$

\subsubsection{Outer nuclear layer and outer plexiform layer}

The outer nuclear layer is composed of the nuclei of the photoreceptors, rods and cones. ${ }^{4}$ Rods function more effectively in low illumination (scotopic vision) and are also sensitive to minimal changes in illumination. ${ }^{3}$ On the other hand, they have less resolving power than cones do and produce a considerably lower visual acuity than cones. ${ }^{3}$

Cones function under high luminance (photopic vision) and are also responsible for color vision and visual acuity. ${ }^{3,4}$ They can rapidly adapt to repeated stimuli but they have low sensitivity to the changes in light intensity. ${ }^{3}$ Cones can be further subdivided into several types by their opsin contents. ${ }^{3}$ Most primates have three types of cones, S, M and L cones. S, M and L cones have the opsins which are sensitive to short (420 nm, blue light), medium (531 nm, green 
light) and long (588 nm, red light) wavelength of light, respectively. ${ }^{3}$ These cones are the basis of trichromatic vision in primates. ${ }^{3}$ Most animals have two types of cones serving dichromatic vision although some have one cone population while some have more than three cone populations. $^{3}$

The processes of the photoreceptors form the outer plexiform layer and synapse on the dendrites of the bipolar and horizontal cells. ${ }^{4}$

\subsubsection{Inner nuclear layer and inner plexiform layer}

The interneurons including bipolar cells, amacrine cells and horizontal cells are located in the INL. ${ }^{4,5}$

Horizontal cells locate in the outermost layer of the INL and laterally connect photoreceptors in the OPL. ${ }^{3}$ Horizontal cells integrate responses from photoreceptors and increase their sensitivity to changes in illumination and color, which is termed lateral inhibition. ${ }^{3}$ Horizontal cells can be divided into two types, HI or luminosity and HII or color. ${ }^{3}$ The HI horizontal cells synapse with both rods and cones. ${ }^{3}$ They respond to light stimuli of any wavelength with graded hyperpolarizations. ${ }^{3}$ The duration and amplitude of the response depends on the intensity and duration of the stimulus. ${ }^{3}$ The HII horizontal cells synapse with cones only. ${ }^{3}$ These cells respond to light stimuli of different wavelength with different polarity. ${ }^{3}$

The dendrites of the bipolar cells synapse with photoreceptors and horizontal cells in OPL and their axons synapse with amacrine cells and RGCs in IPL. ${ }^{3}$ Bipolar cells can be categorized into cone bipolar cells and rod bipolar cells. ${ }^{3}$ Only one type of rod bipolar cells has been described in all mammals. ${ }^{3}$ Because one rod bipolar cells receive input from several rods, the rod pathway is described as a convergent pathway. ${ }^{3}$ At least 10 types of cone bipolar cells 
have been found in human retina. ${ }^{3}$ Diffuse con bipolar cells receive input from more than one cone and thus have a similar convergent pathway as the rod bipolar cells. ${ }^{3}$ At fovea or area centralis, midget bipolar cells synapse only with one cone in a one-to-one relationship and form a nonconvergent pathway. ${ }^{3}$ Bipolar cells can also be categorized based on their responses to light stimuli. ${ }^{3}$ ON-center bipolar cells are depolarized (activated) when the center is stimulated by light, whereas OFF-center bipolar cells are hyperpolarized (inhibited) in response to the light stimulus in the central field. ${ }^{3}$ When a small light stimulate the ON-center bipolar cells and the OFF-surround bipolar cells remain in the dark, the ON-center bipolar cells are depolarized because of the light stimulus and the OFF-surround bipolar cells are also depolarized because of the dark stimulus. ${ }^{3}$ The net result is an enhanced response. ${ }^{3}$ This enhanced response to small, adjacent light and dark stimuli is the cornerstone of high resolution vision. ${ }^{3}$

Amacrine cells locate in the innermost part of the INL. ${ }^{3}$ Amacrine cells can be categorized based on the size, the shape of their dendrites, the neurotransmitter they release, but the most widely used classification is based on the IPL strata in which their dendritic tree is found. ${ }^{3}$ Most amacrine cells are inhibitory neurons containing inhibitory neurotransmitters, GABA or glycine. ${ }^{3}$ Amacrine cells form a complicated network between bipolar cells, RGCs and other types of amacrine cells. ${ }^{3}$ The signal modulation of the amacrine cells increase the sensitivity of RGCs to the changes in illumination, thus contributing to motion, direction and contrast detection. ${ }^{3}$ 


\subsubsection{Retinal ganglion cells}

RGCs are the final output cells of the retina and therefore is critical for vision. ${ }^{3}$ The classifications of RGCs are complicated and can be categorized by both their morphological features and physiological functions. ${ }^{8}$

Midget cells and parasol cells are two types of RGCs in primate which have been examined in detail. ${ }^{8}$ Approximately $80 \%$ of the RGC population in primate retina are midget cells. ${ }^{8}$ They have small dendritic fields, and their axons project to the parvocellular layer of the LGN. ${ }^{8}$ Midget cells have slow axonal conduction, and they respond to chromatic stimuli. ${ }^{8}$ Parasol cells are another type of RGCs which represent about $10 \%$ of the RGC population in primate retina. ${ }^{8}$ The dendritic trees of parasol cells often originate from the thick primary dendrites and then branch out regularly forming a radial-shaped dendritic field. ${ }^{8}$ The axons of parasol cells project to the magnocellular layer of the LGN and the axonal conduction speed is faster than that of midget cells.

In cats, RGCs were first classified as $\alpha$-, $\beta$ - and $\gamma$ - by their morphological features. ${ }^{9,10}$ Alpha-cells have larger somata and dendritic fields, $\beta$-cells have smaller somata and dendritic fields, whereas $\gamma$-cells have small cell bodies with large dendritic fields, and are collectively referred to the non- $\alpha$, non- $\beta$ RGCs. ${ }^{9}$ The electrophysiological responses of RGCs in the cat have been further correlated to the morphological classification and were termed Y-, X- and W-cells, respectively. ${ }^{9,11}$ In cats, $\alpha-, \beta$ - and $\gamma$-cells constitute about $3-5 \%, 45-50 \%$ and $50 \%$ of the feline RGCs, respectively. ${ }^{3}$

The development of advanced technologies, such as intracellular microinjection of the dye into a single RGC has allowed for detailed evaluation of their morphology of the dendritic 
trees and receptive fields. ${ }^{12}$ By this method, 22 subtypes of RGCs have been identified in mouse retina based on their somatic and dendritic features. ${ }^{13}$ In rabbits, 11 subtypes of RGCs were found according to their electrophysiological responses. ${ }^{12}$

In 1998, a new class of RGCs was found. ${ }^{3}$ They have large receptive field, sparse branches, and was shown to project to the hypothalamic suprachiasmatic nucleus (SCN). ${ }^{3}$ Because the SCN regulates the circadian rhythm in mammals, it is believed that these RGCs contributed to regulate the daily behavioral and physiological rhythm by the dark and light cycles. ${ }^{3}$ This new class of RGCs receives input from amacrine cells and cone bipolar cells and also contains a photopigment, melanopsin. ${ }^{3}$ In addition to projecting to SCN, melanopsin RGCs are also thought to project to the parasympathetic oculomotor nucleus and are believed to be involved in control of the pupillary light reflex (PLR). ${ }^{3}$ The existence of melanopsin RGCs may explain the presence of PLR in case of advanced rod-cone degeneration, retinal detachment and other outer retinal disease. ${ }^{3}$

\subsubsection{Axons of RGCs and the nerve fiber layer}

The axons of RGCs form the nerve fiber layer and function as cables transmitting the signals from the final output cells, RGCs, to the brain. ${ }^{14}$ The axons converge at the optic disc and form the optic nerve. ${ }^{7}$ In dogs, the axons are myelinated throughout the entire optic nerve including the optic disc, whereas axons of horses and human are only myelinated after passing through the lamina cribrosa. ${ }^{7}$

Counting the number of axons in the optic nerve provides an alternative way to estimate the RGC population. ${ }^{14}$ Total axon counts have been investigated in numerous species, such as human, ${ }^{15}$ rat, pigeon, ${ }^{16}$ rabbit,${ }^{17}$ eastern chipmunk, ${ }^{18}$ turtle, ${ }^{19}$ beagle dog,${ }^{20}$ dromedary camel ${ }^{21}$ 
and donkey. ${ }^{14}$ Both myelinated and unmyelinated axons were found in those studies and the diameter of myelinated axons was generally larger than that of unmyelinated axons. ${ }^{14,16-19}$ Light and electron microscopy were used in two different pigeon studies to count the numbers of the optic nerves. ${ }^{16,22}$ The discrepancy of the axon count from these two studies was due to the resolution limit of the light microscope. ${ }^{16}$

Osmium tetroxide is a stain which is commonly used to identify the nerve fibers in both peripheral and central nervous system. ${ }^{23}$ Osmium tetroxide is a strong oxidant and reacts with unsaturated double bounds, which are abundant in lipids. Oxidation of lipid double bound degrades the osmium tetroxide and forms the deposition of osmium black. ${ }^{23}$ Therefore, myelin, which is rich in lipids, turns black after immersing in osmium tetroxide. ${ }^{23}$ The advantage of fixation in osmium tetroxide provides sharp myelin staining which enables the identification of the myelin. ${ }^{23}$ The disadvantages of osmium tetroxide include it is a hazardous chemical and it vaporizes readily in room temperature. ${ }^{23}$ In spite of the toxicity of osmium tetroxide, it is a good alternative staining method for paraffin-embedded samples to evaluate the myelinated nerve fibers. $^{23}$

\subsection{Retinal specialization and vision-guided behavior in animals}

The first quantitative RGC topography study was performed by Stone in 1965 with a novel whole-mount technique in a cat. ${ }^{10}$ The result showed a weak, band-shaped, horizontal streak across the retinal meridian and a circular region located temporally with increased RGC density, which is known as area centralis. ${ }^{10}$

Additional retinal neuron topography studies using the same technique have been conducted in other species including horses, ${ }^{24}$ sheep, ${ }^{24} \mathrm{ox},{ }^{24}$ marsupials ${ }^{25}$ and fish. ${ }^{5}$ By 
collectively interpreting the studies of retinal topography from other species, the Terrain Theory was proposed by Hughes in $1977 .{ }^{26}$ It stated that the distribution of RGCs was closely corresponded to the perceived image of the environment. ${ }^{26}$ A horizontal streak is a common finding in terrestrial species whose visual field is not obscured by vegetation, and visual demand is mainly on the horizon. ${ }^{26}$

Three retinal specializations are commonly reported in animals including areae centrales, visual streaks and foveae. ${ }^{5}$

\subsubsection{Areae centrales}

Area centralis is a circular region which contains high density of either RGCs, photoreceptors or both. ${ }^{5}$ It has been identified in numerous vertebrate species, such as cats, dogs and teleost. $^{5}$

The term "area centralis" was first adopted by a German investigator, Chievitz, in 1891to describe the macula lutea in human retina. ${ }^{1}$ The retina in this region was found to be relatively thick with packed retinal neurons. ${ }^{1}$ It was further categorized into area nasalis, area temporalis or area centralis in animals according to the high density region in the retinas. ${ }^{1}$ The locations of the areae centales are also related to the position of the eyes to the head and the degree of binocular vision. ${ }^{1}$ It is mainly found in the temporal retina, and is associated with binocular and acute vision. $^{5}$

Some species, such as the staghorn damselfish (Amblyglyphidodon curacao), possess more than one of areae centrales. ${ }^{1}$ In this species, areae centrales were found in temporal, ventrotemporal and dorso-nasal regions. ${ }^{1}$ The functions of the ventral and ventro-temporal areae centrales are thought to provide frontal, binocular vision and to assist food acquisition, while the 
function of the dorso-nasal area centralis is to negotiate around the projections of the staghorn coral during the escape response of the fish. ${ }^{1}$

\subsubsection{Visual streaks}

According to Terrain Theory, a visual streak is commonly seen in terrestrial species living in the open country whose visual fields are mainly focused on the horizon. ${ }^{1,26}$ Other than the terrestrial species, horizontal streaks are also found in aquatic animals, and are suspected to be beneficial for focusing on the sand-water interface. ${ }^{1}$

The horizontal streak is usually described as a band-shaped region with high density of retinal neurons across the retinal meridian. ${ }^{5}$ In addition to the horizontal streaks, an area centralis is frequently identified at the temporal end of the streak. ${ }^{5}$ The function of this retinal specialization is thought to allow for a panoramic visual field without excessive eye movement which is also critical for predator surveillance. ${ }^{5}$ In the herbivores, in addition to a horizontal streak, there is a conspicuous upward extension of the visual streak in the temporal aspect of the retinas, also referred to as" the anakatabatic area". ${ }^{26,27}$ It was also noticed that the upward extension is particularly longer in taller species than in the shorter species. ${ }^{26}$

The other type of visual streak, a vertical streak, has been reported in only two animal species, two-toed sloths and dromedary camels (Camelus dromedarius). ${ }^{5,21}$ The vertical visual streak in two-toed sloths was believed to be correlated with their behavior of constantly hanging upside down on the trees and observing the environment from sideways. ${ }^{21}$ Harman et al discovered a unique vertical streak on the RGC topography in dromedary camels in $2001 .^{21}$ The adaptive function of the visual streak in dromedary camels is unclear, but was hypothesized to be associated with the flexibility of their necks. ${ }^{21}$ 


\subsubsection{Foveae}

The fovea is an indented area in the retina. ${ }^{5}$ It is an area of high density of photoreceptors and is responsible for acute vision. ${ }^{5}$ The ganglion cells and the interneurons in the inner nuclear layer which receive signals from the photoreceptors in the fovea pit are deviated to the perifoveal region. 5

Two types of foveae have been identified in animals, concaviclivate and convexiclivate. ${ }^{5}$ Convexiclivate foveae have steep slope of the indented wall and is seen in birds and some fishes, whereas concaviclivate foveae are shallow and is seen in monkeys and human. ${ }^{5}$ Some birds, such as predatory birds, possess two foveae, one in the temporal retina and one in the nasal region. ${ }^{5}$ Similar to the function of the temporal area centralis, the function of the temporal fovea is to aid in the detection of trivial movement in the visual field, while the nasal fovea provides monocular vision and enhances acuity by magnifying the image. ${ }^{5}$

\subsection{Visual acuity}

Visual acuity represents the maximal resolving power of the eye. ${ }^{28}$ In human, the visual acuity can be examined by reading a Snellen chart which involves with the visualization of the letters on the chart and then interpreting them in the central nervous system in conjunction with verbal elaboration. ${ }^{28}$ The visual acuity of humans is usually expressed in diopter or the comparison to an emmetropic person, such as 20/20 represents emmetropia (normal sightedness) and 20/40 represents myopia (near sightedness). ${ }^{28}$

Visual acuity has been examined in numerous species, such as primates, ${ }^{29}$ eagles, ${ }^{30}$ rabbits, ${ }^{31}$ cats, ${ }^{32-35}$ and horses ${ }^{36,37}$ and the results were significantly different among species. Several approaches were introduced to examine the visual acuity in animals including behavioral 
test, estimation by ocular parameter and electrical potential recording evoked from retinal neurons and the visual cortex. ${ }^{38}$

The visual acuity of animals can be expressed in two ways, cycles per degree or minute of arc. ${ }^{28}$ When visual acuity is expressed in cycles per degree, one cycle contains a black bar and a white bar. ${ }^{28}$ The numbers of cycles within one degree of visual angle is the result of visual acuity. ${ }^{28}$ The more cycles an animal can see in one degree of visual angle, the higher visual acuity this animal has. On the contrary, the other expression, minute of arc, describes the angles in minutes subtended by a black or white bar. The lower in minutes an animal can resolve, the higher visual acuity this animal has.

Visual acuity can be influenced by optical and neural factors, ${ }^{28}$ and it is also highly correlated to visual ecology and behavior ecology in animals. ${ }^{1}$ Optical factors, such as the luminance level, refractive power of the corneas and lenses and the size of pupils, significantly influence the visual acuity by changing the amount of light delivered to the retina. ${ }^{28}$ Neural limitation of the visual acuity is determined by the distribution and characteristic of photoreceptors and RGCs and the convergence of the photoreceptors on the RGCs. ${ }^{1,38}$

\subsubsection{The behavioral test}

The behavioral methods to evaluate visual acuity have been performed in the cat, ${ }^{32,33,39}$ rabbit, ${ }^{31}$ horse,${ }^{36,37}$ rhesus monkey and squirrel monkey. ${ }^{29}$ Before the tests, the animals were trained to give a repetitive response when detecting a stimulus. In one cat study, the cats were taught to drink while seeing a safe stimulus and to stop drinking while seeing a warning stimulus. ${ }^{33}$ After training, the animals were tested with different frequency of gratings as the stimulus. ${ }^{33}$ By repeating the tests several times, the upper threshold at which the animals could 
see the stimulus as individual bars was obtained and was interpreted as the best estimation of visual acuity when all the animals failed to react correctly. ${ }^{33}$

A similar study was performed in horses by Timney $e t a l^{37}$ in 1992 and Harmen $e t a l^{36}$ in 1999. The visual acuity in horses was reported to be 15 to 25 cycles per degree ${ }^{37}$ and 16.5 cycles per degree ${ }^{36}$, respectively. The behavioral approach may reflect the closest visual acuity in animals, but several limitations were reported in the studies including the learning ability and cooperation of the animals, the luminance level and the distance of the grating. ${ }^{36,37}$

\subsubsection{Approach by ocular anatomical parameter}

By measuring the focal length of the eye and its RGC density, it is possible to estimate the upper limit, or cut-off limit, of visual acuity in animals by sampling theorem. ${ }^{38}$ In species whose convergence of cone to RGC is close to a one-to-one ratio, measuring the density of cones could be another method to estimate the visual acuity. ${ }^{38}$

The estimation of visual acuity from the peak RGC density in cats showed similar results as the estimation from behavioral test and cortical visual-evoked potential recording. 33,35,38,40 This method requires the measurement of posterior nodal distance (PND), the distance between the center of the lens to the retina, and the peak RGC or cone density. ${ }^{38}$ The PND was reported to be approximate 0.67 of the axial length of the globe in diurnal animals and 0.51 to 0.54 in nocturnal animals. ${ }^{38}$ After obtaining PND, retinal magnification factor (RMF), the distance on the retina per visual degree, can be calculated by the following equation: $2 \pi \mathrm{PND} / 360 .{ }^{38} \mathrm{RMF}$ represents the magnification extent of the image on the retina. ${ }^{38}$

Two main theories of sampling array have been developed for estimating visual acuity by ocular anatomical parameters. 
The first theory proposed by Pettigrew et al in $1988^{38}$ stated that the sampling array at peak RGC density region was organized in a square lattice. The linear density (cells/mm) is estimated by calculating the square root of the peak RGC density (cells $/ \mathrm{mm}^{2}$ ). Two cells are required to distinguish one cycle of a given spatial frequency; therefore, the linear density is divided by two. Then this value is multiplied by RMF to obtain the maximal visual acuity at the peak RGC region. Equation: $0.5 \times \mathrm{RMF} \times[\sqrt{ }($ peak $\mathrm{RGC}$ density $)] / 2$

The second theory was extrapolated from a theory proposed by Williams and Coletta in $1987 .{ }^{41}$ It suggested that the photoreceptor arrangement at the high density area is in a hexagonal or triangular lattice. The theory was hypothesized to be applicable on RGC arrangement. In this method, the maximal visual acuity is estimated by the equation: $0.5 \times \mathrm{RMF} \times[2 \times($ peak $\mathrm{RGC}$ density) $/ \sqrt{ } 3]^{1 / 2}$.

\subsubsection{Approach by electrophysiological methods}

Measuring the evoked electrical potential to estimate the visual acuity in animals can be performed by analyzing the responses from either the retinal neurons or the visual cortex. ${ }^{42}$ In the study of measuring electrical potential evoked from visual cortex in cat, an electrode was placed in contact with the dura above the visual cortex via an opening on the skull. ${ }^{35,42}$ The cat was tested with gratings in different frequencies while it was alert ${ }^{35}$ or anesthetized. ${ }^{42}$ The results from measuring the electrical potential of the visual cortex in a conscious cat were in agreement with the estimation of visual acuity from behavioral tests. ${ }^{35}$

The pattern electroretinogram (PERG) has been used to measure the visual acuity in dogs, cats and human because the PERG electrical potential is generated in the inner retinal layers, 
primarily in the ganglion cell layer (GCL). ${ }^{43,44}$ The result of PERG recordings correlated well with the result of Snellen resolution in human and behavioral tests in animals. ${ }^{44}$

A similar experimental setting of measuring evoked electrical potential of the occipital lobe in the cat was applied to dogs. The potentials from both visual cortex and PERG were recorded in dogs and the results of visual acuity in dogs were in agreement with each other. ${ }^{43}$

\subsection{Techniques for analyzing RGC topography}

Numerous techniques have been developed to study the retinal cell distribution including retrograde labeling, immunohistochemical labeling and cresyl violet staining. ${ }^{45}$

\subsubsection{Retrograde labeling of retinal ganglion cells}

Positive identification of RGCs is based on the fact that the axons of the RGCs form the nerve fiber layer of the retina, exit the eye at the optic nerve head and gather to form the optic nerve. ${ }^{46}$ Thus, retrograde labeling of RGCs is a technique that takes advantage of this unique characteristic. $^{47}$ In general, after injecting or applying retrograde labeling dye to the retinorecipient zones of the brain or to the end of optic nerve stalk, the dye is taken up by the axons and is transported back to the RGC somata by axoplasmic flow. ${ }^{47}$

Several neural tracers have been used, such as horseradish peroxidase, ${ }^{48}$ fluorogold, ${ }^{49}$ Lucifer Yellow $^{50}$ and DiI (1,1'-dioctadecyl-3,3,3',3'-tetramethylindocarbocaynine perchlorate), ${ }^{51}$ in animal models to reveal the dendritic trees, morphology and the distribution of the RGCs. Successful ex vivo retrograde labeling to reveal RGC topography has been reported in rodents, ${ }^{47}$ cats $^{50}$ and New Zealand white rabbits ${ }^{50}$ by applying Lucifer Yellow and DiI to the optic nerve stalk of the enucleated eyes. 


\subsubsection{DiI}

DiI is a lipophilic, orange to red fluorescent dye with a peak emission wavelength at 563

nm. ${ }^{52}$ It has been widely used in retrograde labeling of RGCs in both living and fixed tissues. ${ }^{53}$ DiI is transported by lateral diffusion of the cell membrane after it is applied at the optic nerve stalk. ${ }^{53}$ In a study performed on living chick and rats, the transport rate of DiI was about 6 $\mathrm{mm} /$ day due to active dye transport process. ${ }^{53}$ In the fixed tissue, the rate decreased to 0.4 $\mathrm{mm} / \mathrm{day}^{53}$

\subsubsection{Lucifer Yellow}

Lucifer Yellow $\mathrm{CH}$ dilithium is a yellow fluorescent dye with the peak emission wavelength at $540 \mathrm{~nm} .{ }^{47}$ Lucifer Yellow (LY) has been used broadly in the intracellular dyeinjection technique to demonstrate the dendrites of the RGCs. ${ }^{12,47}$ It is known that LY is impermeable to gap junctions and harmless to the cells. ${ }^{47}$ Therefore, it is also a good dye for retrograde labeling. ${ }^{47}$ In several studies LY was applied to the end of the optic nerve stalk to label RGCs in the mouse ${ }^{47}$ and New Zealand white rabbit. ${ }^{12}$ After applying LY at the optic nerve stalk, it was taken up by the transected optic nerve fiber and then was transported back to RGCs by the axoplasmic flow. ${ }^{47,50}$

In the mouse study, $3 \% \mathrm{LY}$ and $8 \%$ neurobiotin was applied at the optic nerve stalk and the eyecup was immersed in oxygenated Ames medium for 40 minutes at room temperature. ${ }^{47}$ Ten-minute dark and light cycle was used during the incubation period. This technique achieved successful labeling of RGC somata and also revealed the dendritic trees and axons of RGCs. ${ }^{47}$ In the study of cats and New Zealand white rabbits, a plastic tube was placed to cover the optic nerve stalk and the tube was filled with $6 \% \mathrm{LY} .{ }^{50}$ Then the eyecups were incubated in the 
oxygenated Ames medium for 4 to 24 hours. Successful demonstration of RGC dendrites was reported. ${ }^{50}$ However, no transport rate of LY has been reported in the literature.

\subsubsection{Immunohistochemical labeling}

\subsubsection{General concept of immunohistochemistry}

Immunohistochemistry is a technique using the specificity between antibodies and antigens to reveal a certain population of cells or tissues. ${ }^{54}$ In order to label the target cells, a specific antibody for the target cells (antigens) must be identified first. ${ }^{54}$

Antibodies can be classified into two main categories, monoclonal and polyclonal antibodies. ${ }^{54}$ Polyclonal antibodies are produced by several immune cells, whereas monoclonal antibodies are produced by a single cell line. ${ }^{54}$ In the manufacture of monoclonal antibody, the desired cell line is developed by fusing a mammalian immune cell with a tumor cell. This results in endless replication of antibodies. ${ }^{54}$

The pros of using polyclonal antibody in immunohistochemistry include the low expense of producing antibody and large production of the antibody in a short period of time. ${ }^{54}$ The cons of polyclonal antibodies are that the production of non-specific binding antibodies which create background signals may occur. On the other hand, monoclonal antibodies are very specific to an epitope, but the expense of developing a cell line or an animal model to produce monoclonal antibodies is high. ${ }^{54}$

There are two main types of labels on the antibodies, enzymes or fluorophores. ${ }^{54}$ The enzyme-labeled antibodies are used with secondary antibodies or (strept)avidin. The enzymelabeled antibodies can be visualized with a brightfield light microscope after triggering the 
chromogenic reaction. The fluorophore-labeled antibodies have to be evaluated with a fluorescent microscope with a proper setting of excitation and emission wavelength. ${ }^{54}$

\subsubsection{Retinal ganglion cell markers and the Brn-3 family}

Several RGC markers have been used to label the RGCs in rats, mice and rabbits for different purposes of study, such as Thy $1,{ }^{55} \mathrm{AB} 5^{56,57}$ and Brn-3a. ${ }^{49,58}$ Thy-1 is a surface glycoprotein expressed largely in RGCs. ${ }^{55}$ In the healthy retina, Thy-1 expression was limited to the RGCs and their axons. ${ }^{55}$ However, in the damaged retinal tissues, Thy-1 expression in RGCs was down-regulated, but up-regulated in Müller cells. ${ }^{59-61}$ AB5 is a monoclonal RGC marker which labeled both RGCs and their axons. ${ }^{56}$ AB5 has shown reliable RGC labeling in rabbits, ${ }^{56}$ hamster $^{57}$ and cats. ${ }^{56}$

The Brn-3 family consists of three family members, Brn-3a, Brn-3b and Brn-3c. ${ }^{58}$ The Brn-3 family is a transcription factor expressed from the POU domain gene. ${ }^{58}$ In monkey, rabbit, cat and chicken retinas, antibodies against all three Brn-3 members showed immunoreactivity mainly in GCL. ${ }^{58}$ Occasional labeling was found in the cells of the inner plexiform layer or inner nuclear layer which were presumably displaced RGCs. ${ }^{58}$ In the mouse retina, anti-Brn-3a, antiBrn-3b and anti- Brn-3c antibodies all labeled a subset of RGCs, while anti-Brn-3c antibodies labeled fewer RGCs than the other two. ${ }^{58}$ In the same study, RGCs labeled by anti-Brn-3a and anti-Brn-3b antibodies largely overlapped with each other in mouse retina. ${ }^{58}$

In the cat retina, strong immunoreactivity was found in $70 \%$ of $\gamma$-cells with anti-Brn-3a antibody labeling and weak immunoreactivity in all $\alpha$-cells and about $70 \%$ of $\beta$-cells. ${ }^{58}$ By contrast, anti-Brn-3b labeling was uniform in intensity, indicating a similar level of expression of Brn-3b in all $\alpha$-, $\beta$ - and $\gamma$-cells. ${ }^{58}$ However, anti-Brn-3c only labeled about $50 \%$ of $\gamma$-cells 
without showing any immunoreactivity in $\alpha$ - and $\beta$-cells. ${ }^{58}$ In macaques, nearly all of the RGCs which were positive for anti-Brn-3a and anti-Brn-3a antibodies labeling were also labeled by a retrograde neural tracer. ${ }^{58}$

In a rat study, anti-Brn-3a showed reliable labeling of RGCs in both normal retinas and optic nerve-injured retinas. ${ }^{49}$ It also reported that $92.2 \%$ of retrograde neural tracer, fluorogold, positive RGCs also co-labeled with anti-Brn-3a antibody. ${ }^{49}$

Although immunohistochemistry has been applied in numerous animal species, there is no immunohistochemical study reported in alpaca retinas.

\subsubsection{Cresyl violet staining}

Nissl substance is the rough endoplasmic reticulum of the neurons in the central nervous system, and it has been reported to be abundant in retinal neurons. ${ }^{45}$ Theoretically, staining for Nissl substances allows for the identification of RGCs so as to differentiate them from other neuroglial cells or non-neural elements which also reside in the GCL. ${ }^{45}$ Nissl staining, also known as cresyl violet staining, has been broadly employed in the studies of retinal ganglion cell topography in various species including cats, ${ }^{10,40}$ rabbits, ${ }^{62}$ horses, ${ }^{24,36}$ and sheep. ${ }^{24}$

Due to the difficulty of distinguishing small RGCs from other small retinal neurons, Stone proposed a comparative method to analyze the morphology of RGCs in cats. ${ }^{45}$ In this method, the optic nerve was severed to induce RGC apoptosis, and the cell population in those retinas was devoid of RGCs several months later. Then a normal retina and a retina with RGC apoptosis from the same species were compared. Based on this method, RGCs were identified as the cells which were present in the normal retina but absent in the retina from an eye with severed optic nerve. The common feature of this cell population included eccentric, palely 
stained nuclei with distinct nucleoli. The cytoplasm had clumped darkly-stained Nissl substances. ${ }^{45}$

In spite of the accessibility of Nissl staining, it has been debatable whether or not the morphologic features of the RGCs stained with cresyl violet are reliable to differentiate them from displaced amacrine cells or glial cells in the ganglion cell layer (GCL). ${ }^{45}$ Thus, one would be prone to overestimate the total RGC count. ${ }^{48}$ Although overestimation of RGC has been found in Nissl staining, the patterns of RGC distribution, in terms of the high density area, established by Nissl staining and retrograde labeling were not different in a study of the harlequin tusk fish. ${ }^{48}$ 


\section{STATEMENT OF HYPOTHESIS}

The RGC topography has been evaluated in numerous mammalian species, but there has been no study performed on alpaca retinas. It is unknown whether or not alpacas have a unique vertical streak of dense RGCs as reported in dromedary camels. There is also a paucity of information about the visual acuity in alpacas.

The overall objective of this study was to investigate the RGC distribution and the visual acuity in alpacas. The hypothesis of the study was that alpacas have similar RGC distributions as do dromedary camels rather than that of other grazing animals.

In order to examine this hypothesis, three RGC labeling methods were used to stain alpaca retinas, retrograde labeling of Lucifer Yellow, immunohistochemical labeling using antiBrn-3a antibody and cresyl violet staining. Axon counting was performed to compare the number of axons with the result of RGC labeling. The visual acuity of alpacas was estimated by the ocular parameter method. 


\section{MATERIALS AND METHODS}

\subsection{Sample acquisition}

Five eyes (three right eyes and two left eyes) from four male alpacas between two- and three-year-old weighing between 61 and $68 \mathrm{~kg}$ and were included in the study. Pre-mortem ophthalmic examinations which consisted of menace responses, pupillary light reflexes, Schirmer tear tests, fluorescent staining, applanation tonometry, slit lamp biomicroscopy and indirect funduscopy were performed on all animals. The results were within normal limits. All animals were euthanized with intravenous injection of pentobarbital sodium (Beuthanasia ${ }^{\circledR}$-D Special; Intervet Inc., Summit, NJ, USA; $85 \mathrm{mg} / \mathrm{kg}$ ) for unrelated medical problems. Globes with optic nerve attached were collected immediately after euthanasia. Axial length of the globes, the distance between the anterior and posterior poles of the globe, were measured $(n=3)$ with a Vernier caliper and recorded prior to the dissection. Globes were dissected into eyecups by removing the anterior segments, lenses and partial vitreous. Four samples received retrograde labeling and one sample did not receive retrograde labeling. Then the eyecups were immersion fixed in $4 \%$ paraformaldehyde in phosphate-buffered saline (PBS, $\mathrm{pH} 7.4,0.1 \mathrm{M}$ phosphate) for one hour. The fixation process was terminated by washing three times in PBS. All protocols were approved by Colorado State University Institutional Animal Care and Use Committee (Protocol number: 12-3324A).

\subsection{Labeling and staining techniques of RGCs}

3.2.1 Retrograde labeling of the retinal ganglion cells

A 4.7-mm or 5.3-mm inner diameter vinyl plastic tube was placed at the end of optic nerve stalk and glue (Super Glue ${ }^{\circledR}$, Rancho Cucamonga, CA) was applied around the edge of 
plastic to enhance the adherence of the plastic tube to the sclera $(n=4) .3 \%$ Lucifer Yellow $\mathrm{CH}$ dilithium powder (L0259, Sigma-Aldrich, Saint Louis, MO) in Ames medium (A1420, SigmaAldrich, Saint Louis, MO) was instilled into the plastic tube and the opening of the tube was sealed with clay. The entire eyecup was immersed in oxygenated Ames medium at room temperature. 10-minute dark and light cycles were conducted throughout the entire LY labeling period. Oxygenated Ames medium was changed every 30 minutes. The period of LY labeling was four hours for eyecups from Alpaca 1and Alpaca 2 and eight hours for the eyecups from Alpaca 3. No LY labeling was performed on the eyes from Alpaca 4. After LY labeling, the plastic tube was removed and the optic nerve was rinsed three times in PBS. Then the eyecups were fixed in $4 \%$ paraformaldehyde for one hour and the fixation process was terminated by washing in PBS three times.

\subsubsection{Tissue preparation for immunohistochemical study}

For the control study, a small, ventral portion of each retina with attached sclera (approximate $2 \mathrm{~mm}$ from ora serrate and $5 \mathrm{~mm}$ in width) from each sample was cryoprotected in $30 \%$ sucrose. The samples were placed in the optimal cutting temperature compound (TissueTek® O.C.T. compound, Sakura Finetek, Torrance, CA) in a plastic cup. Then the samples were frozen in $-20^{\circ} \mathrm{C}$ and $-80^{\circ} \mathrm{C}$ refrigerators for 15 and 20 minutes, respectively. A cryostat microtome was used to cut the samples into cross-sectioned retinas.

Prior to the retinal extraction, the optic nerve stalks were removed from the eye cups. The retinas were retracted from the eye cups after removing scleras and ciliary bodies and were completely separated after cutting at the optic disc by a scalpel blade. Then the retinas were dissected into six portions including temporodorsal, temporomiddle, temporoventral, nasodorsal, 
nasomiddle and nasoventral as illustrated in Figure 1. The landmarks of each retinal portion including shapes, notches, vessels and pigments were recorded for orientation. In order to facilitate the removal of the vitreal residue, each retinal portion was treated with type IV-S bovine testicular hyaluronidase (2120 units/retina; H3884; Sigma-Aldrich, Saint Louis, MO) for two hours and the activity of bovine testicular hyaluronidase was terminated by washing in PBS three times.

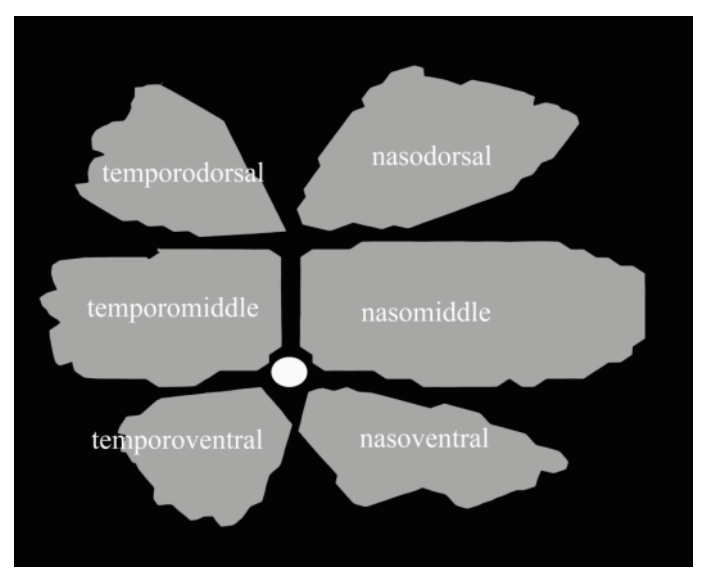

Figure 1 The retinal dissection after extracting from the eyecup. A midline section was made first and each half of the retinas was dissected into dorsal, middle and ventral regions.

3.2.3 Antibodies

The primary and secondary antibodies used in the present study are listed in Table 1.

Table 1 Primary and secondary antibodies

\begin{tabular}{|l|l|l|l|}
\hline Primary antibodies & Serum & Source & Catalog number \\
\hline Anti-Brn-3a antibody & Goat & $\begin{array}{l}\text { Santa Cruz } \\
\text { Biotechnology }\end{array}$ & sc31984 \\
\hline $\begin{array}{l}\text { Anti-Lucifer Yellow } \\
\text { antibody }\end{array}$ & Rabbit & Life technologies & A-5750 \\
\hline $\begin{array}{l}\text { Secondary antibodies } \\
\text { (host animal, antiserum) }\end{array}$ & $\begin{array}{l}\text { Fluorophore } \\
\text { (excitation/emmision) }\end{array}$ & Source & Catalog number \\
\hline Donkey, anti-goat & $\begin{array}{l}\text { AlexaFluor } \\
(495 / 519)\end{array}$ & Life technologies & A11055 \\
\hline Donkey, anti-goat & $\mathrm{Cy3}^{(\circledR)}(550 / 570)$ & Millipore & AP180C \\
\hline Donkey, anti-rabbit & $\mathrm{Cy3}^{(\circledR}(550 / 570)$ & Millipore & AP182C \\
\hline
\end{tabular}


3.2.4 Study for Lucifer Yellow labeling and anti-Lucifer Yellow antibody on cross-sectioned retina

3.2.4.1 Evaluating retrograde labeling of Lucifer Yellow on cross sectioned retina

The cross-sectioned retinas were treated with 1: 200 diluted goat anti-Brn-3a antibody (sc31984; Santa Cruz Biotechnology, Santa Cruz, CA) and 1:400 diluted donkey anti-goat Cy3 ${ }^{\circledR}$ secondary antibody (AP180C; Millipore, Billerica, MA). Cy $3{ }^{\circledR}$ secondary antibody was used to avoid the crosstalk of the fluorescent from AlexaFluor ${ }^{\circledR} 488$ (Alexa Fluor ${ }^{\circledR} 488$ Dye; A11055; Life technologies, Grand Island, NY) and LY.

3.2.4.2 Evaluating retrograde labeling of Lucifer Yellow with anti-Lucifer Yellow antibody on cross-sectioned retina

The cross-sectioned retinas were treated with 1:200 diluted goat anti-Brn-3a antibody, 1:100, 1:200, 1:300, 1:400 and 1:500 diluted rabbit anti-LY antibody (A-5750; Life technologies, Grand Island, NY), 1:400 diluted donkey anti-goat AlexaFluor ${ }^{\circledR} 488$ secondary antibody and 1:400 diluted donkey anti-rabbit Cy $3^{\circledR}$ secondary antibody (AP182C; Millipore, Billerica, MA). Anti-LY antibody was used because no LY fluorescent was detected in the peripheral retina.

3.2.5 Immunohistochemical labeling of retinal ganglion cells with anti-Brn3a antibody

\subsubsection{Whole-mounted retinas}

The retinas $(\mathrm{n}=5)$ were first blocked in $1 \%$ bovine serum in PBS with $0.3 \%$ Triton-X 100 for two hours and then incubated in 1:100 diluted goat anti-Brn3a antibody for 24 hours. After washing in PBS, the samples were next immersed in 1:400 diluted donkey anti-goat AlexaFluor ${ }^{\circledR}$ 488 secondary antibody and a nuclear marker, TO-PRO ${ }^{\circledR} 3$ (1:5000; T3605; Life technologies, 
Grand Island, NY), in blocking solution for two hours, followed by PBS washes. The retinas were then whole-mounted on microscope slides with GCL facing up. Fluorescent mounting medium (H-1000; Vectashield ${ }^{\circledR}$ Mounting Medium; Vector Laboratories, Burlingame, CA) was applied and the specimens were coverslipped.

\subsubsection{Cross-sectioned retinas}

The positive controls for the study was performed on either alpaca or wild-type rat retinal sections using both goat anti-Brn3a antibody and donkey anti-goat AlexaFluor ${ }^{\circledR} 488$ secondary antibody with a nuclear marker. The negative control was treated similarly, but the anti-Brn3a antibody was omitted.

\subsubsection{Cresyl violet staining of retinal ganglion cells}

Samples labeled with anti-Brn-3a antibody were also used for cresyl violet staining. Before cresyl violet staining, the samples were washed in PBS overnight to remove the fluorescent mounting medium. Then the samples were dried on the microscope slides for staining.

Two drying methods were used:

\section{Method 1: heat dry}

The samples were rinsed in PBS and then mounted onto the microscope slides with GCL facing up. The samples were fixed by formalin vapor in a metal box with a covered lid on a hot plate for 30 to 60 minutes. Then the samples were left in the metal box overnight.

\section{Method 2: air dry}


The samples were rinsed in PBS and then mounted onto the microscope slides with GCL facing up. The samples were air-dried in a metal box which was partially covered by a lid for 60 minutes.

The protocol of cresyl violet staining was modified from a previously described method ${ }^{48}$ as shown in Table 2. The solutions were prepared as the formula in Table 3. After the cresyl violet staining, the samples were coverslipped with a permanent mounting medium $\left(\mathrm{MM} 24^{\circledR}\right.$; 3801122, Leica Biosystem, Buffalo Grove, IL). The functions of xylene and ethanol are to defat and dehydrate the sample. Differential solution was used to clear the excessive staining solution.

Table 2 Cresyl violet staining protocol

\begin{tabular}{|l|l|l|l|l|l|}
\hline Step & Staining tray & time & Step & Staining tray & time \\
\hline 1 & $100 \%$ xylene & 10 minutes & 11 & $20 \%$ ethanol & 30 seconds \\
\hline 2 & $100 \%$ xylene & 10 minutes & 12 & $70 \%$ ethanol & 30 seconds \\
\hline 3 & $100 \%$ ethanol & 5 minutes & 13 & $95 \%$ ethanol & 30 seconds \\
\hline 4 & $100 \%$ ethanol & 2 minutes & 14 & differential solution & 1 minute \\
\hline 5 & $95 \%$ ethanol & 2 minutes & 15 & $100 \%$ ethanol & 1 minute \\
\hline 6 & $70 \%$ ethanol & 2 minutes & 16 & $100 \%$ ethanol & 1 minute \\
\hline 7 & $20 \%$ ethanol & 2 minutes & 17 & $100 \%$ xylene & 2 minutes \\
\hline 8 & distilled water & 2 minutes & 18 & $100 \%$ xylene & 2 minutes \\
\hline 9 & $0.1 \%$ cresyl violet & 20 minutes & $\begin{array}{l}\text { Xylene }(8400-1, \text { StatLab Medical Product, } \\
\text { McKinney, TX) }\end{array}$ \\
\hline 10 & distilled water & quick rinse & $\begin{array}{l}100 \% \text { Ethanol (3803686, Leica Biosystem, Buffalo } \\
\text { Grove, IL) }\end{array}$ \\
\hline
\end{tabular}

Table 3 Solution preparation

$\underline{0.1 \% \text { cresyl violet solution (filter the solution before use) }}$

0.2 g cresyl violet acetate powder (C5042, Sigma-Aldrich, Saint Louis, MO)

$5 \mathrm{ml}$ glacial acetic acid (A490-212, Thermo Fisher Scientific Inc., Pittsburgh, PA)

$5 \mathrm{ml} 1.0 \mathrm{M}$ sodium acetate anhydrous (Molecular weight: 82.03; S210-2, Thermo Fisher

Scientific Inc., Pittsburgh, PA)

$190 \mathrm{ml}$ of distilled water

Differential solution

$900 \mathrm{ml} \mathrm{95 \%} \mathrm{ethanol}$

$1 \mathrm{ml}$ glacial acetic acid 
Because of less than optimal staining result, several steps of cresyl violet protocol were modified which included a shorter incubation time (1 minute, 30 seconds and 15 seconds) in differential solution, skipping the initial defatting and dehydrating processes in xylene and ethanol (step 1 to 7), different rinse solution (distilled water, PBS and tap water), different $\mathrm{pH}$ of cresyl violet solutions ( $\mathrm{pH}=3.3$ and 3.6). A different cresyl violet protocol extrapolated from one used by Dr. Eric Storey at Louisiana State University was also tried (Table 4).

Table 4 The cresyl violet staining protocol from Dr. Storey at LSU

\begin{tabular}{|l|l|l|l|l|l|}
\hline Step & Staining tray & time & Step & Staining tray & time \\
\hline 1 & Distilled water & Rinse & 11 & $70 \%$ ethanol & 2 minutes \\
\hline 2 & $70 \%$ ethanol & 2 minutes & 12 & Distilled water & 2 minutes \\
\hline 3 & $95 \%$ ethanol & 2 minutes & 13 & $0.1 \%$ cresyl violet & 8 minutes \\
\hline 4 & $100 \%$ ethanol & 2 minutes & 14 & $70 \%$ ethanol & 20 seconds \\
\hline 5 & $100 \%$ ethanol & 2 minutes & 15 & $95 \%$ ethanol & 30 seconds \\
\hline 6 & $100 \%$ xylene & 6 hours & 16 & $100 \%$ ethanol & 1 minute \\
\hline 7 & $100 \%$ xylene & overnight & 17 & $100 \%$ ethanol & 1 minute \\
\hline 8 & $100 \%$ ethanol & 2 minutes & 18 & $100 \%$ xylene & 1 minute \\
\hline 9 & $100 \%$ ethanol & 2 minutes & 19 & $100 \%$ xylene & 1 minute \\
\hline 10 & $95 \%$ ethanol & 2 minutes & \multicolumn{5}{|l}{} \\
\hline
\end{tabular}

3.3 Osmication of the myelin of optic nerve fiber

The connective tissue around the optic nerve stalk was removed. A one- to two-mm thick, cross-sectioned optic nerve specimen $(\mathrm{n}=1)$ was incubated in $4 \%$ osmium tetroxide (Stevens Metallurgical Corp., New York, NY) overnight and was paraffin embedded. Three- $\mu$ m thick sections were cut with a microtome for analyses.

\subsection{Image acquisition}

3.4.1 Study for Lucifer Yellow labeling and anti-Lucifer Yellow antibody on cross-sectioned retinas 


\subsubsection{Evaluating retrograde labeling of Lucifer Yellow on cross sectioned retina}

The images of the cross-sectioned retinas with LY retrograde labeling was taken by a Zeiss LSM 510 confocal laser microscope (Carl Zeiss, Oberkochen, Germany) controlled by computer software program (Zeiss LSM Image Examiner, Thornwood, NY) with a 40× objective. A fluorescent microscope equipped with a computer-driven motorized stage (Ludl Electronic Products Ltd., Hawthorne, NY) and a camera (AxioCam, Carl Zeiss, Thornwood, NY) controlled by the computer software program (Axiovision, Carl Zeiss, Thornwood, NY) was also used to evaluate the fluorescent of LY.

3.4.1.2 Evaluating retrograde labeling of Lucifer Yellow with anti-Lucifer Yellow antibody on cross-sectioned retina

The same confocal microscope was used to capture the images of cross-sectioned retina with a $40 \times$ objective to evaluate the labeling of anti-LY antibody and anti-Brn-3a antibody.

\subsubsection{Immunohistochemical labeling of retinal ganglion cells with anti-Brn3a antibody}

\subsubsection{Whole-mounted retinas}

The methods for image acquisition and analysis were modified from a previously described method. ${ }^{6}$ Images were taken with the same aforementioned fluorescent microscope. For whole-mounted retinas, the entire retinal portion was first scanned with a 10× objective in the manner of multi-tile acquisition (MosaicX, Axiovision, Carl Zeiss, Thornwood, NY) and the tiles were obtained apposing each other without overlapping. Each tile was manually focused and images were captured with a $20 \times$ objective. Images taken with a confocal laser microscopy of 
whole-mounted retinas were used to demonstrate the co-labeling of anti-Brn3a antibody and nuclear marker, ToPro3.

\subsubsection{Cross-sectioned retinas}

For the control study, the same fluorescent microscope used for the whole-mounted retinas study was used to acquire the images with a $20 \times$ objective. In addition, several images were taken with a $40 \times$ objective with a confocal laser microscope to show the co-labeling of antiBrn3a antibody and the nuclear marker.

\subsubsection{Cresyl violet staining of retinal ganglion cells}

Images were taken with a 100× objective with a software-controlled (Axiovision, Carl Zeiss, Thornwood, NY) light microscope (Carl Zeiss, Thornwood, NY) which was equipped with a computer-driven motorized stage (Ludl Electronic Products Ltd., Hawthorne, NY) and a camera (AxioCam, Carl Zeiss, Thornwood, NY).

\subsubsection{Osmication of the myelin}

The cross-sectioned optic nerve specimen was scanned with the same light microscope used for cresyl violet staining. The specimen was initially scanned with a $10 \times$ objective in the manner of multi-tile acquisition without tile overlapping. Each tile was manually focused and images were captured with a $100 \times$ objective.

\subsection{Image analysis}

3.5.1 Study for Lucifer Yellow labeling and anti-Lucifer Yellow antibody on cross-sectioned retinas 
3.5.1.1 Evaluating retrograde labeling of Lucifer Yellow on cross sectioned retina

Images taken with confocal microscope were evaluated using computer software (Zeiss

LSM Image Examiner, Thornwood, NY) and processed with computer software (Photoshop 12.0, Adobe Systems, Inc., San Jose, CA).

3.4.1.2 Evaluating retrograde labeling of Lucifer Yellow with anti-Lucifer Yellow antibody on cross-sectioned retina

Images taken with the confocal microscope were evaluated with computer software (Zeiss LSM Image Examiner, Thornwood, NY) and processed by computer software (Photoshop 12.0, Adobe Systems, Inc., San Jose, CA).

3.5.2 Immunohistochemical labeling of retinal ganglion cells with anti-Brn-3a antibody

\subsubsection{Whole-mounted retina}

The RGC counting was performed by either semi-automatic counting or manual labeling of the anti-Brn3a positive cells using a computer software program (Image-Pro Plus 6.0 for Windows; MediaCybernetics, Rockville, MD). A sequential filter setting was modified from a previously described method $^{6}$ and was applied on the images for semi-automatic counting in order to extract the cell edges from the background and assist in the RGC counting.

A flatten enhancement filter was used to balance out the background IpLSFltApply(LF_EDGEPL,50,50,2,5) and the images were converted to 8-bit grey scale images (IpWsConvertImage(IMC_GRAY, CONV_SCALE, 0, 0, 0, 0)). Then an edge+ large spectral filter (IpLSFltShow(0)) was used to enhance the cell edges. Three passes of the median enhancement filter $(\operatorname{IpFltMedian}(7,3))$ were used to remove the background noise. The 
processed images were counted according to their nuclear size range. The cells with nuclear sizes between 17 to $115 \mu \mathrm{m}^{2}$ were included in the count. This number was pre-determined by measuring the nuclear size from 16 randomly-selected areas with different cell density.

The function of watershed split or split objective in the software (Image-Pro Plus 6.0 for Windows; MediaCybernetics, Rockville, MD) was selected to separate the individual cells with the presence of cell overlapping on the original fluorescent images. The grey-scaled images were compared to the original fluorescent image to exclude the debris by manually unselecting the

counting. The counting results were documented on a spreadsheet (Microsoft Office Excel 2010, Redmond, WA) and the estimated RGC density was calculated for reconstructed retinal map.

\subsubsection{Cross-sectioned reitnas}

For the control retinas, images were evaluated using the fluorescent microscope. In addition, several images from the confocal microscope were scanned with computer software (Zeiss LSM Image Examiner, Thornwood, NY) and processed by computer software (Photoshop 12.0, Adobe Systems, Inc., San Jose, CA).

3.5.3 Cresyl violet staining of retinal ganglion cells

Images taken with the light microscope was evaluated with computer software (Axiovision, Carl Zeiss, Thornwood, NY).

3.5.4 Osmication of the myelin of the optic nerve fiber

The axon counting was performed manually and the axons were digitally numbered with computer software (Image-Pro Plus 6.0 for Windows; MediaCybernetics, Rockville, MD). The 
results were entered into a spreadsheet (Microsoft Office Excel 2010, Redmond, WA) and the estimated total axon number was calculated.

\subsection{Reconstructed retinal map}

After completing the RGC counting, a topographical RGC map was made by computer software (OriginPro 8.1, Northampton, MA) according to the orientation recording. The reconstructed images were then further processed by computer software (Photoshop 12.0, Adobe Systems, Inc., San Jose, CA).

3.7 Estimation of visual acuity by ocular parameter

Two ocular parameters are required to estimate visual acuity, posterior nodal distance (PND) and peak RGC density. Based on two theories of RGC arrangement, two equations were used to estimate the visual acuity accordingly.

Equation based on RGC arrangement in square lattice: $0.5 \times \mathrm{RMF} \times[\sqrt{ }($ peak RGC density $)] / 2$ Equation based on RGC arrangement in hexagonal lattice: $0.5 \times \mathrm{RMF} \times[2 \mathrm{x}$ (peak RGC density) $/ \sqrt{ } 3]^{1 / 2}$

\subsection{Statistical analyses}

The results of five eyes are presented with descriptive statistics by computer software (Microsoft Office Excel 2010, Redmond, WA). No formal statistical analysis was performed due to small sample size. Medians were used to represent as the parameter. 


\section{RESULT}

4.1 Labeling and staining techniques of retinal ganglion cells

4.1.1 Study for Lucifer Yellow labeling and anti-Lucifer Yellow antibody on cross-sectioned retinas

4.1.1.1 Evaluating retrograde labeling of Lucifer Yellow on cross-sectioned retina

The images taken with either confocal microscope or fluorescent microscope showed no labeling of RGC with LY of the peripheral retina in figure 2.

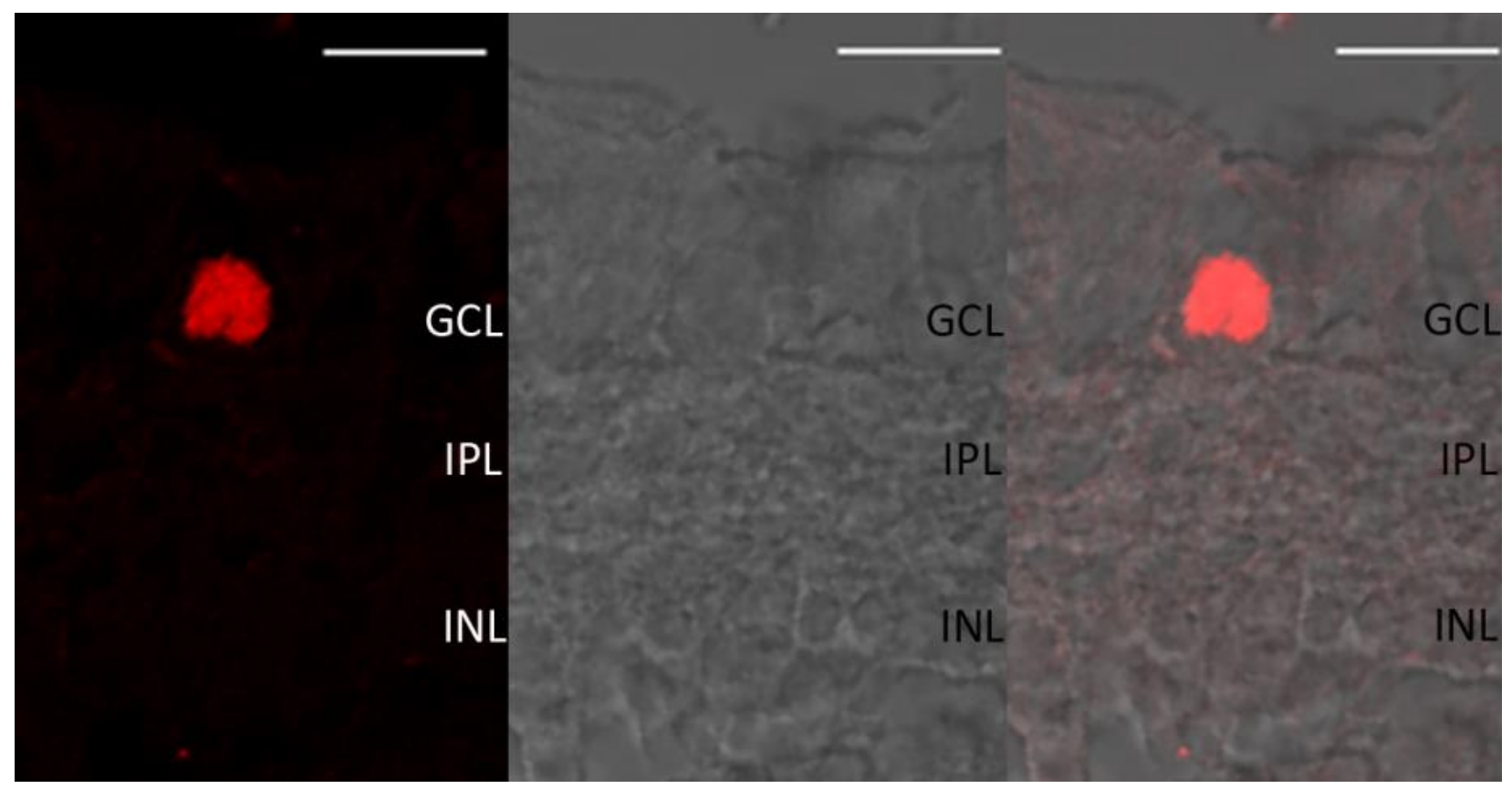

Figure 2 Positive labeling of RGCs with anti-Brn-3a (red) in GCL but no Lucifer Yellow labeling. Image was acquired by a confocal laser microscope. The left image was fluorescent labeling of anti-Brn-3a antibody. The middle image was the bright field image and the right was the merged image. Scale bar: $20 \mu \mathrm{m}$ 
4.1.1.2 Evaluating retrograde labeling of Lucifer Yellow with anti-Lucifer Yellow antibody on cross-sectioned retina

The images taken with a confocal microscope showed positive labeling of the RGC with anti-Brn-3a antibody in GCL and diffuse, faint labeling of anti-LY in GCL, inner plexiform layer (IPL) and INL as illustrated in figure 3.

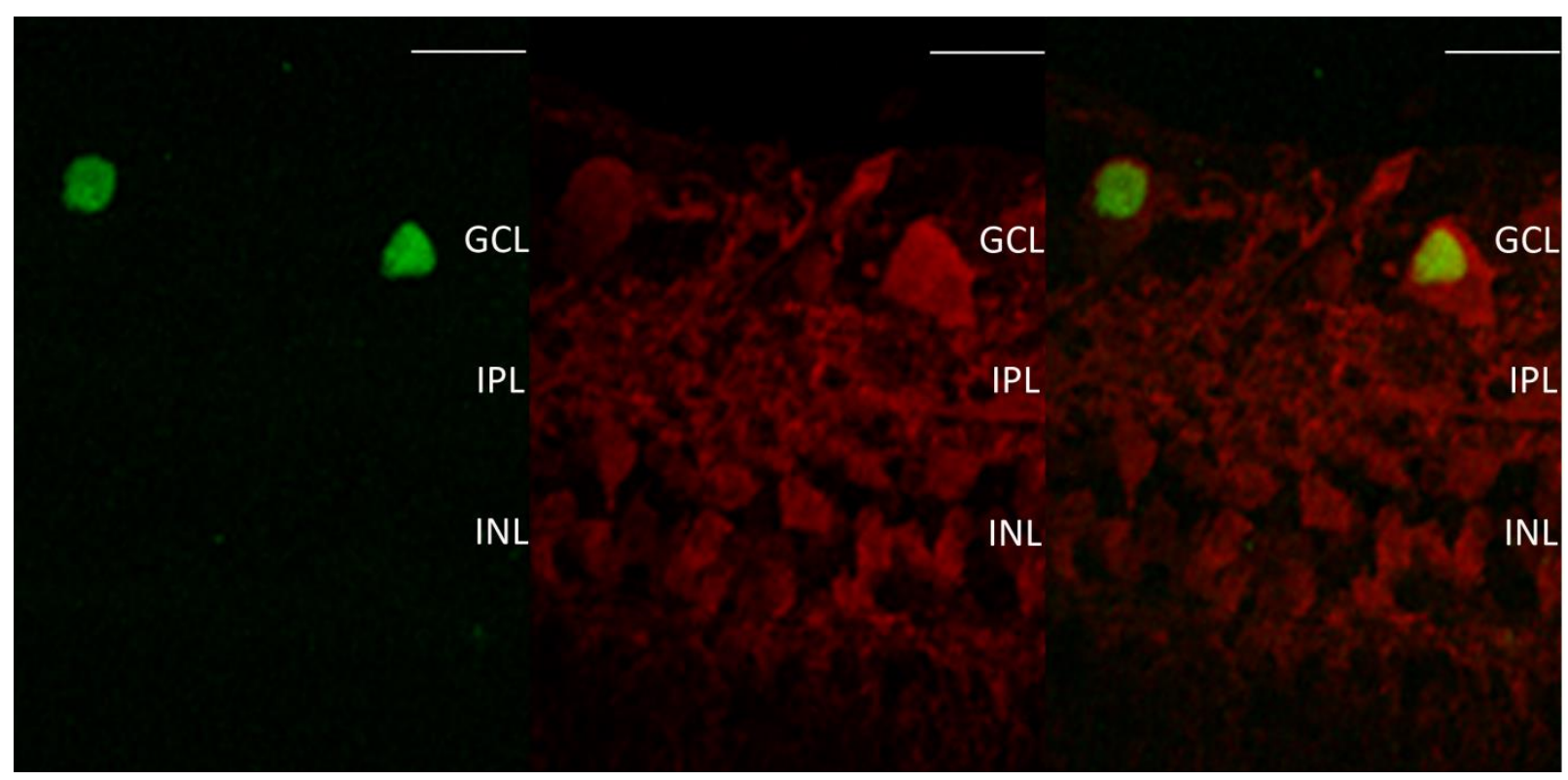

Figure 3 Positive labeling of RGC with anti-Brn-3a (green) in GCL with diffuse, faint labeling of anti-LY (red) was in GCL, inner plexiform layer (IPL) and INL. The concentration of anti-LY antibody in this figure was 1:100. Image was acquired by a confocal laser. The left image was fluorescent labeling of anti-Brn-3a antibody. The middle image was fluorescent labeling of antiLY and the right was the merged image. Scale bar: $20 \mu \mathrm{m}$ 
4.1.2 Immunohistochemical labeling of retinal ganglion cells with anti-Brn3a antibody

\subsubsection{Whole-mounted retinas}

Figure 4 shows the image of an entire retina which was scanned with computer software (MosaicX, Axiovision, Carl Zeiss, Thornwood, NY) and processed by computer software (Photoshop 12.0, Adobe Systems, Inc., San Jose, CA). Figures 5 and 6 shows the images acquired with a fluorescent microscope from two areas with high and low density of RGCs, respectively. Figure 7 shows a confocal microscopic image of the GCL with both anti-Brn-3a antibody and nuclear marker labeling. In figure 7, several cells in GCL were Brn-3a positive and some were Brn-3a negative. 

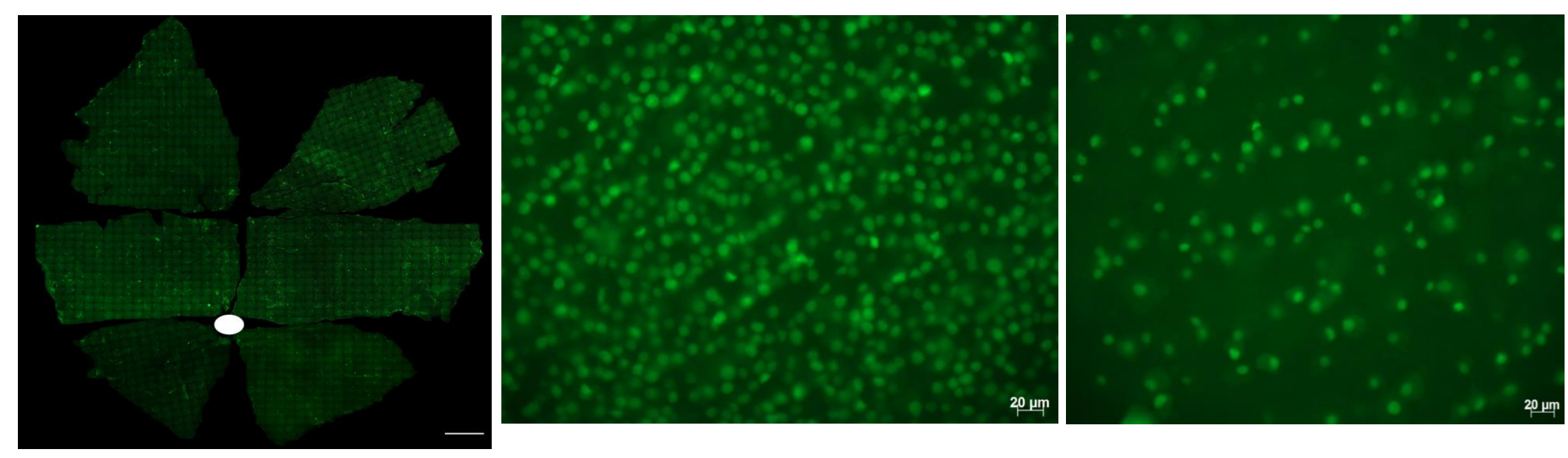

Figure 4 The retina scanned with computer software (MosaicX, Axiovision, Carl Zeiss, Thornwood, NY) and processed by computer software (Photoshop 12.0, Adobe Systems, Inc., San Jose, CA). The white dot represents the location of optic disc. Scale Bar: $3.5 \mathrm{~mm}$
Figure 5 A retinal area with a high density of Brn3a positive cells in GCL. Image was acquired by a fluorescent microscope.
Figure 6 A retinal area with low density of Brn3a positive cells in GCL. Image was acquired by a fluorescent microscope. 


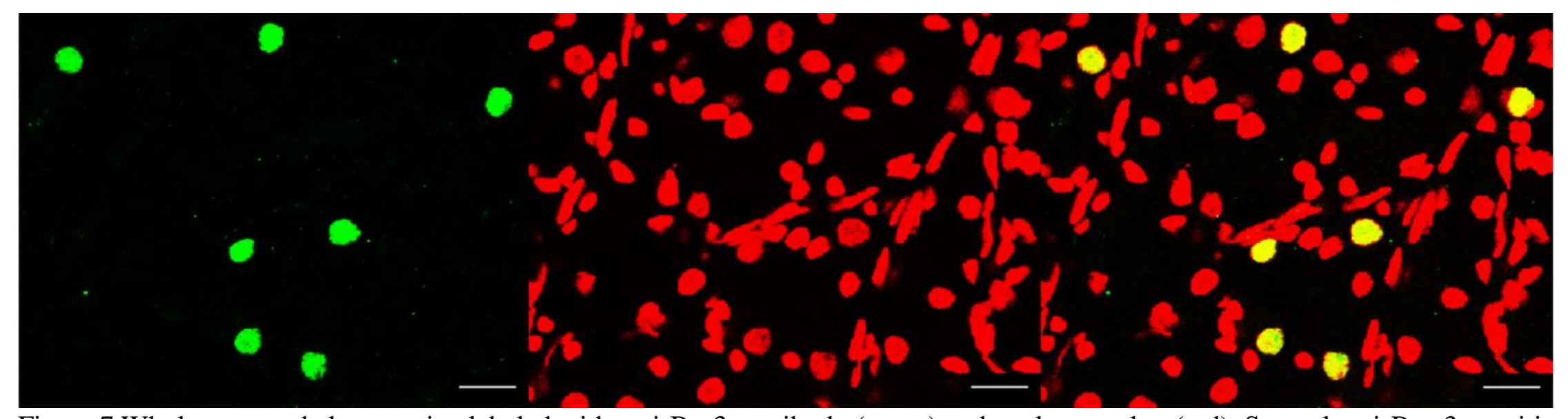

Figure 7 Whole-mounted alpaca retina labeled with anti-Brn3a antibody (green) and nuclear marker (red). Several anti-Brn-3a positive RGCs were identified in the GCL on a whole-mounted alpaca retina. Image was acquired by a confocal laser microscope. The left image was fluorescent labeling of anti-Brn-3a antibody. The middle image was fluorescent labeling of ToPro- 3 and the right was the merged image. Scale bar: $20 \mu \mathrm{m}$ 


\subsubsection{Cross-sectioned retinas}

The images showed several cells in the GCL and the INL with positive labeling of anti-Brn-3a antibody as illustrated in figure 8. There were other cells in the GCL which were Brn-3a negative.

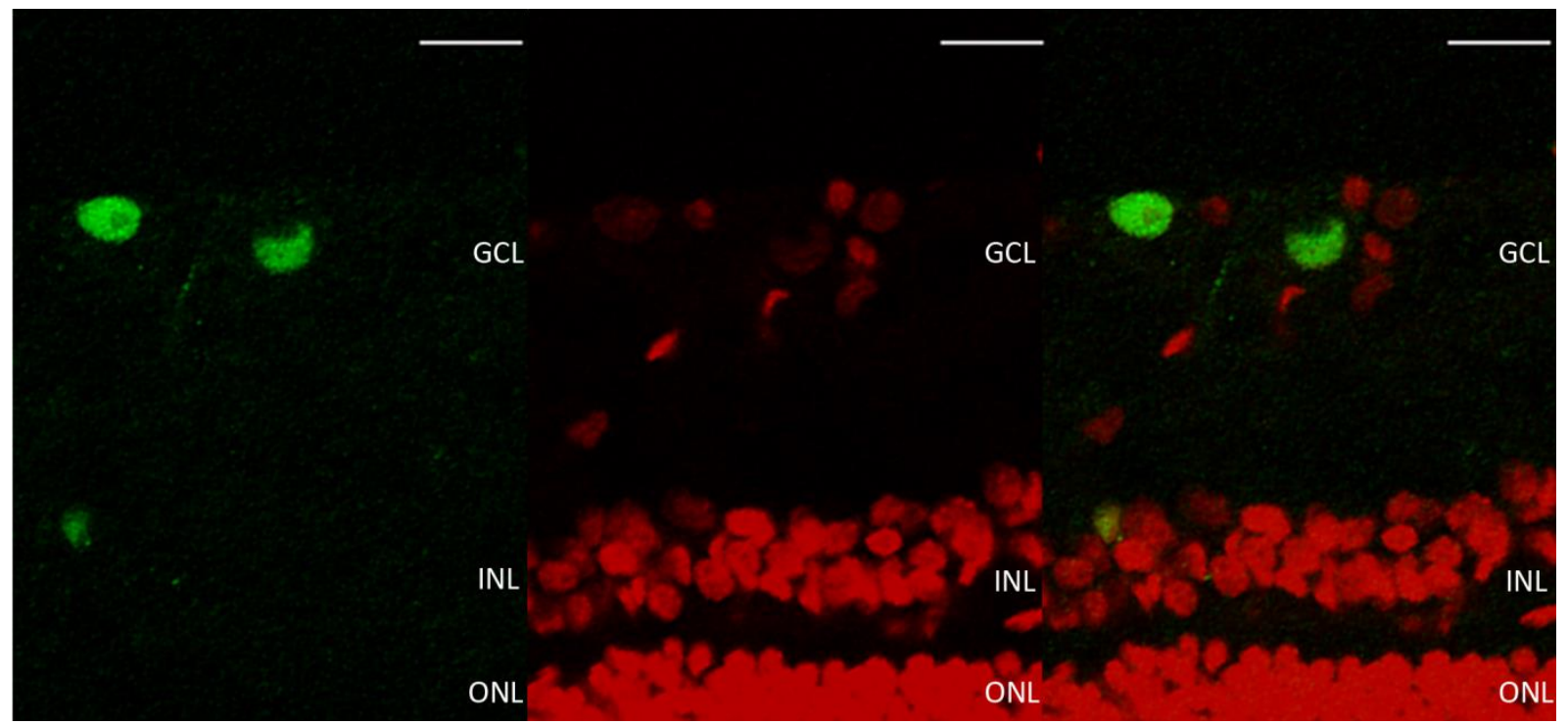

Figure 8 Positive labeling of RGCs with anti-Brn-3a antibody (green) with a nuclear marker, TOPRO3 (red), in the GCL and INL. Image was acquired by a confocal laser microscope. The left image was fluorescent labeling of anti-Brn-3a antibody. The middle image was fluorescent labeling of ToPro-3 and the right was the merged image. Scale bar: $20 \mu \mathrm{m}$ 


\subsubsection{Cresyl violet staining}

No Nissl substance was found in the images taken with the light microscope with a 100x objective.

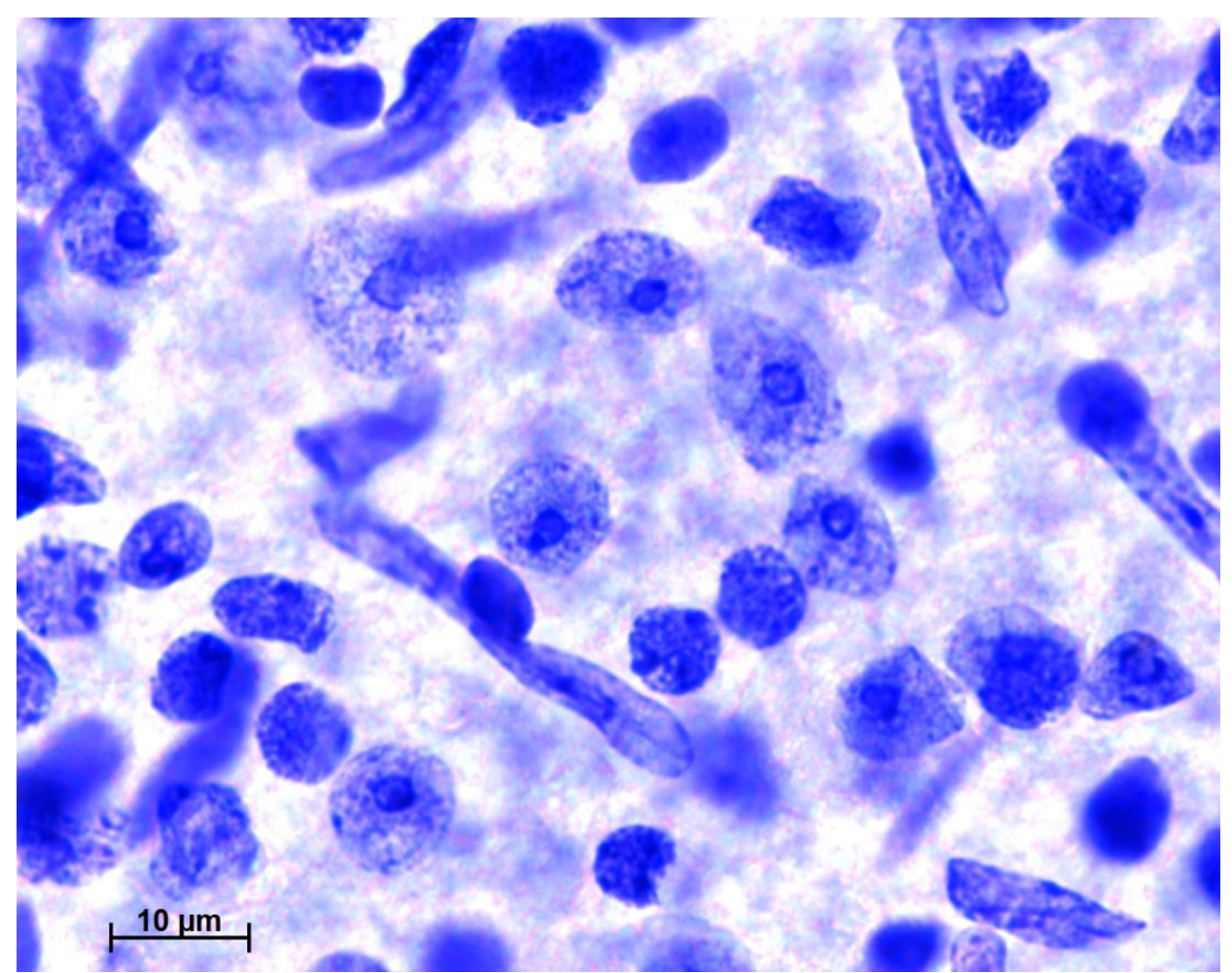

Figure 9 Cresyl violet staining of the cells in GCL. No Nissl substance was found in the cells in GCL. Image was acquired by a light microscope. 
4.2 Osmication of the myelin of the optic nerve fiber

The cross-sectioned area of the optic nerve was $6.17 \mathrm{~mm}^{2}(\mathrm{n}=1)$ and the result of the axon counting was $675,188(n=1)$. Figure 10 showed the image of the cross-sectioned optic nerve acquired with computer software (MosaicX, Axiovision, Carl Zeiss, Thornwood, NY) and processed by computer software (Photoshop 12.0, Adobe Systems, Inc., San Jose, CA). Figure 11 showed an area of optic nerve sampled for axon counting.

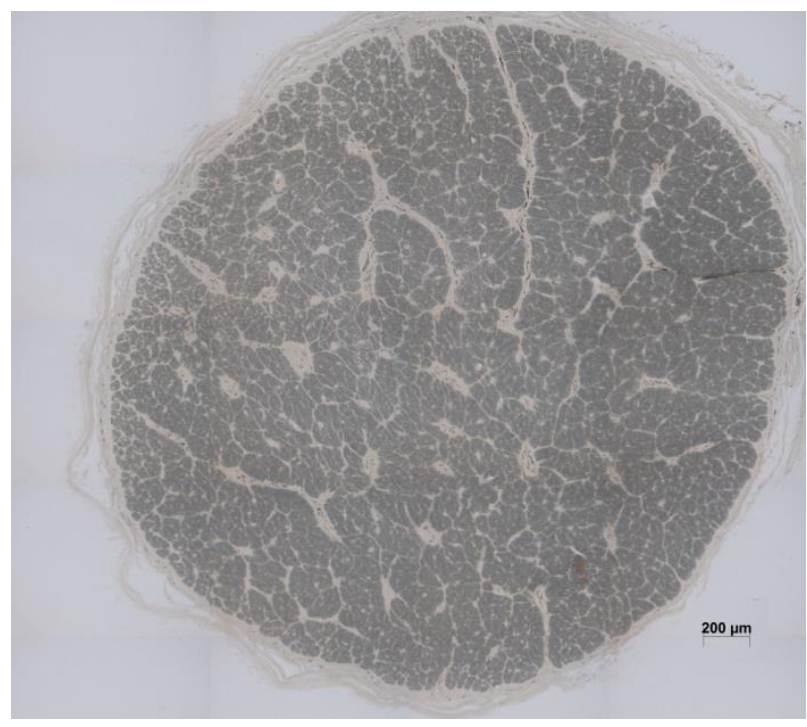

Figure 10 The cross-sectioned optic nerve scanned by computer software (MosaicX, Axiovision, Carl Zeiss, Thornwood, NY) and processed by computer software (Photoshop 12.0, Adobe Systems, Inc., San Jose, CA).

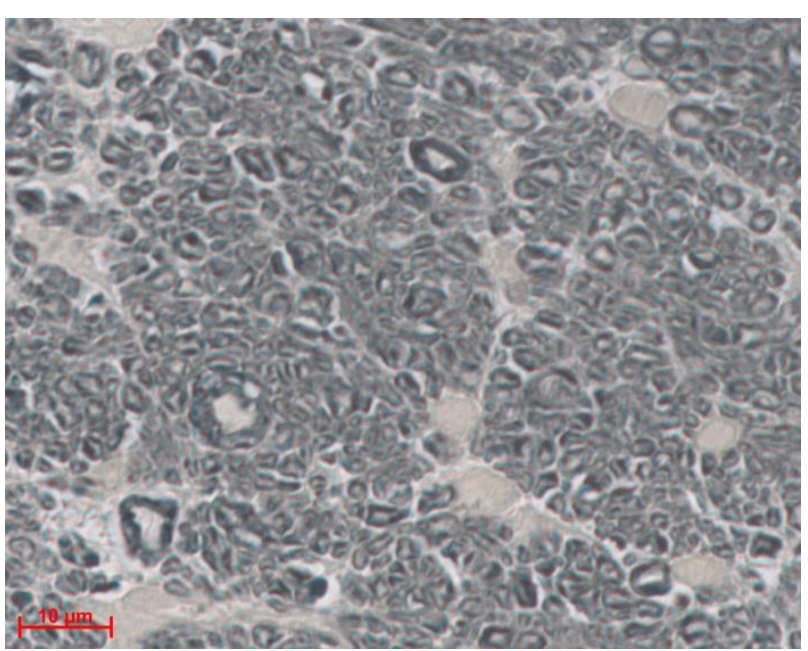

Figure 11 An area sampled for axon counting. The image was taken by a light microscope. 


\subsection{Reconstructed retinal map}

The reconstructed retinal maps from the five eyes are illustrated in Figure 12. The maps showed a distinct, ax-shaped horizontal streak superior to the optic nerve head with a temporal upward extension in all five samples. The highest RGC density regions were all in the temporal retinas.
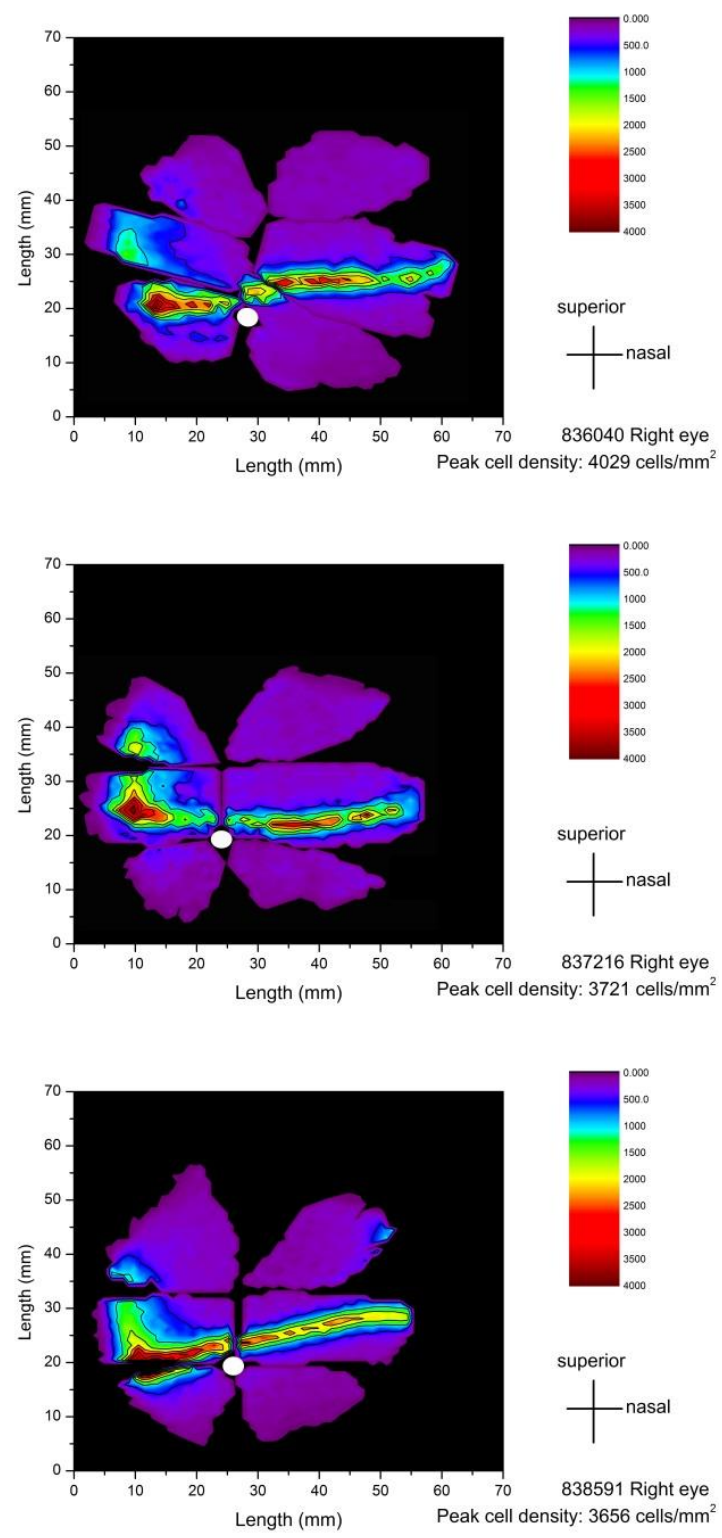
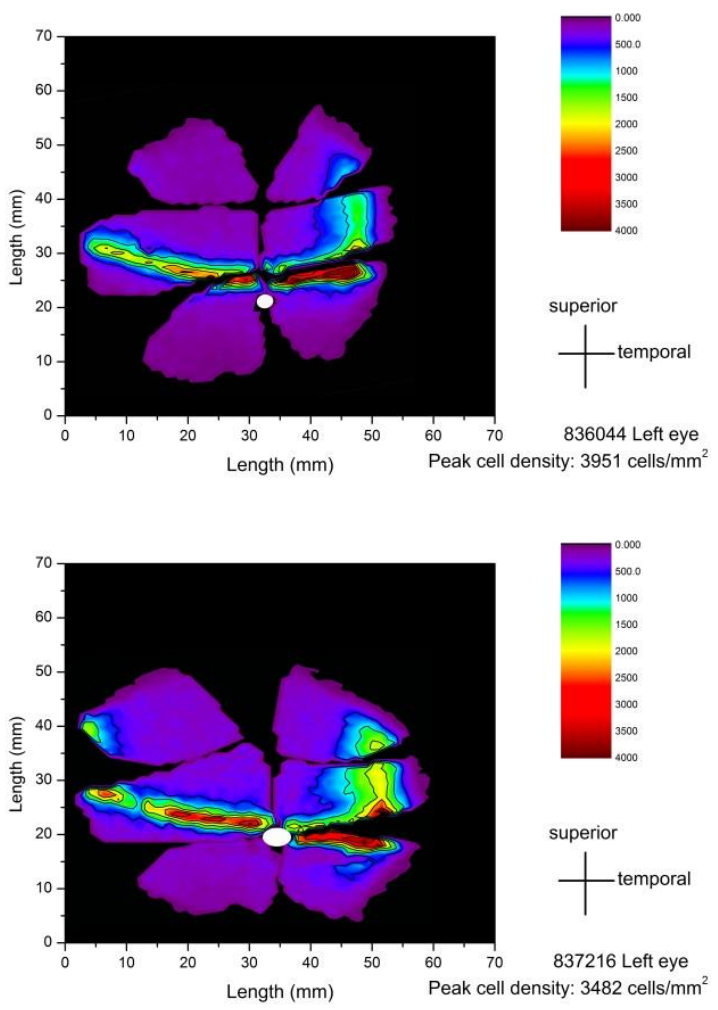

Figure 12 The reconstructed retinal maps of all five samples. The result shows a distinct, axshaped horizontal streak of high RGC density superior to the optic nerve head with a temporal, upward extension. 


\subsection{Estimation of visual acuity by ocular parameter}

Retinal dimensions were estimated from the scanned retinal images by summing up the area of the scanned tiles. The median of retinal dimensions was $883.8 \mathrm{~mm}^{2}(\mathrm{n}=5$, range between 811.6 and $\left.954.2 \mathrm{~mm}^{2}\right)$ and the median of the sampled area for counting was $207.4 \mathrm{~mm}^{2}(24 \%$, $\mathrm{n}=5$, range between 166.4 and $226.9 \mathrm{~mm}^{2}$ ) as summarized in Table 5. In the whole-mounted technique, the retinas were composed of the tiles scanned with a $10 \times$ objective and the images representing each of the tiles were captured with a $20 \times$ objective. Thus, the estimated total RGC count per retina was obtained by multiplying the number of Brn3a-positive cells in each tile image captured with a $20 \times$ objective by four. The median of the estimated total RGC count per eye was 408,322 cells ( $n=5$, range between 342,512 and 502,028 cells) and the median of the peak RGC density was 3,721 cell $/ \mathrm{mm}^{2}$ ( $\mathrm{n}=5$, range between 3,482 and 4,029 cell $/ \mathrm{mm}^{2}$ ). 
Table 5 A summary of results of retinal dimension, sampled area, estimated RGC count, peak RGC density, axial length of the globe, PND, RMF and estimated visual acuity of all five samples

\begin{tabular}{|c|c|c|c|c|c|c|c|c|c|c|c|}
\hline $\begin{array}{c}\text { Case } \\
\text { Number }\end{array}$ & Eye & $\begin{array}{c}\text { Retinal } \\
\text { dimension } \\
\left(\mathrm{mm}^{2}\right)\end{array}$ & $\begin{array}{c}\text { Sampled } \\
\text { area }(\mathrm{mm})\end{array}$ & $\begin{array}{c}\text { Sampled area } \\
(\%)\end{array}$ & $\begin{array}{l}\text { Estimated total } \\
\text { RGC count } \\
\text { (cells/ retina) }\end{array}$ & $\begin{array}{c}\text { Peak RGC } \\
\text { density } \\
\left(\text { cell } / \mathrm{mm}^{2}\right)\end{array}$ & $\begin{array}{l}\text { Axial } \\
\text { length of } \\
\text { the globe } \\
(\mathrm{mm})\end{array}$ & $\begin{array}{l}\text { PND } \\
(\mathrm{mm})\end{array}$ & $\begin{array}{c}\text { RMF } \\
\text { (mm/degree) }\end{array}$ & $\begin{array}{l}\text { Visual acuity } \\
\text { by theory } 1 * \\
\text { (cycle/degree) }\end{array}$ & $\begin{array}{l}\text { Visual acuity } \\
\text { by theory } 2 \dagger \\
\text { (cycle/degree) }\end{array}$ \\
\hline Alpaca 1 & $\mathrm{R}$ & 811.6 & 166.4 & 21 & 342,512 & 4,029 & N/A & N/A & N/A & N/A & N/A \\
\hline Alpaca 2 & $\mathrm{~L}$ & 954.2 & 224.2 & 24 & 408,322 & 3,951 & N/A & N/A & N/A & N/A & N/A \\
\hline Alpaca 3 & $\mathrm{R}$ & 883.8 & 196.6 & 22 & 396,192 & 3,721 & 35 & 23.5 & 0.4 & 12.5 & 13.4 \\
\hline Alpaca 3 & $\mathrm{~L}$ & 945.4 & 226.9 & 24 & 502,028 & 3,482 & 37 & 24.8 & 0.4 & 12.8 & 13.7 \\
\hline Alpaca 4 & $\mathrm{R}$ & 864.2 & 207.4 & 24 & 431,148 & 3,656 & 34 & 22.8 & 0.4 & 12.4 & 12.9 \\
\hline Mean \pm SD & & $891.8 \pm 59.2$ & $204.3 \pm 24.6$ & $22.9 \pm 1.5$ & $416,040 \pm 58,056$ & $3,767 \pm 222$ & $35.3 \pm 1.5$ & $23.7 \pm 1.0$ & $0.4 \pm 0.0$ & $12.4 \pm 0.4$ & $13.3 \pm 0.4$ \\
\hline Median & & 883.8 & 207.4 & 24 & 408,322 & 3,721 & 35 & 23.5 & 0.4 & 12.5 & 13.4 \\
\hline
\end{tabular}

PND: posterior nodal distance; RMF: retinal magnification factor; N/A: data not obtained

*Theory 1: sampling array in square lattice

$\dagger$ Theory 2: sampling array in hexagonal latti 


\section{DISCUSSION}

\subsection{RGC topography and visual behavior in alpacas}

Results of the present study showed that alpacas have a distinct, ax-shaped horizontal streak of high RGC density superior to the optic nerve head with a temporal, upward extension. This RGC topography is different from the vertical streak reported in dromedary camels. Compared to the RGC maps in other species, the RGC topography in alpacas is very similar to other grazing animals, such as horses, sheep and ox. ${ }^{24} \mathrm{~A}$ horizontal streak has been found in a plethora of mammalian species including both nocturnal and diurnal animals, and a horizontal streak with an upward, temporal extension has been exclusively identified in herbivores. ${ }^{26}$ Additionally, the extension is much greater in taller species than in the shorter species. ${ }^{26}$

It has been postulated that the function of the horizontal streak in herbivores is to assist with predator surveillance in open country so that the animal does not need excessive eye and head movement. ${ }^{5,26}$ The perception to the movement of either prey or predator is decreased with the presence of the visual streak. ${ }^{5}$ Alpacas evolved in the Andean highlands in South America and have been domesticated from other wild camelid species for more than six thousand years. ${ }^{63}$ In spite of the difference in the landscape between Andean highlands and the natural habitats of horses, sheep and ox, it is likely that the horizontal streak in alpacas helps with acquiring food and guarding herds as well.

The reason for the striking differences in RGC topography in dromedary camels and in alpacas is unknown. The differences could reflect the variation in the natural habitats between desert and Andean highlands, the visual behavior, or both. On the other hand, the method used by Harman $e t l^{21}$ to analyze the RGC topography of dromedary camel was different from the 
techniques used in the present study. In the dromedary camel study, ${ }^{21}$ the RGCs were stained with cresyl violet. The sampled area for RGC counting was only $1 \%$ of the retinal dimension and the sample number was three eyes, compared to $21-24 \%$ in the present study. In order to minimize the sampling error and misinterpretation of RGCs, a reliable RGC marker, anti-Brn-3a antibody, was applied on five healthy retinas in the present study. Increasing sampling area and using a reliable RGC marker in the current study should lead to more accurate RGC counts.

\subsection{Visual acuity}

Visual acuity in animals has been investigated in primates ${ }^{29}$, eagles, ${ }^{30}$ rabbits,${ }^{31}$ cats, ${ }^{32,33}$ and horses. ${ }^{36,37}$ Not surprisingly, primates and birds have the highest visual acuity which is needed for foraging. ${ }^{30}$ In dromedary camels, the highest visual acuity estimated by peak RGC density was 10.4 cycles per degree in temporal retina, which was slightly lower than that in horses. ${ }^{21}$ This degree of visual acuity was thought to be associated with a relatively complex herd society in horses. ${ }^{21}$

Several methods for estimating visual acuity in animals have been described in the literature including behavioral tests, electrophysiological recordings, eye movement or response to stimuli and ocular anatomical parameters. ${ }^{38}$ Visual acuity calculated from peak RGC densities is considered as an upper limit of the ability of an animal to distinguish fine details and it has been shown that the visual acuity of an animal correlates well with the results of behavioral testing. ${ }^{38}$ Visual acuity in cats estimated by peak RGC densities has closely agreed with the results of behavioral test and evoked cortical potentials. ${ }^{32,33,35}$ Similar correlations were demonstrated in the studies of visual acuity in horses as well. ${ }^{36,37}$ Therefore, the results reported in the present study were deemed to be a satisfactory estimate of visual acuity in alpacas. 
The maximal visual acuity of alpacas reported in the present study was in the temporal retina. It was calculated to be either 12.4 or 13.3 cycles per degree according to the two approaches in the sampling theorem. The results suggest that visual acuity in alpacas is slightly higher than that in dromedary (10.4 cycles/degree) and Bactrian camels (10 cycles/degree) ${ }^{21}$ but lower than that of horses (16.5 cycles/degree). ${ }^{36,37}$

\subsection{RGCs and displaced RGCs in alpacas}

RGCs can be classified into several categories according to their morphology and electrophysiological function. Intracellular microinjection or retrograde labeling of the neural tracers can be used to reveal the dendritic field and dendritic branches of single RGCs. ${ }^{12,64}$ However, because of the inability to label the RGCs with LY in the present study, the classification of RGCs was not possible.

In figure 8, several Brn3a-positive cells were found in the inner nuclear layer, which are most likely to be displaced RGCs (dRGCs). Displaced RGCs have been reported in several species, such as mouse, ${ }^{47}$ Australian lungfish ${ }^{65}$ and monkey. ${ }^{66}$ The population of the dRGC was reported to be about $2 \%$ of the total RGC in the mouse ${ }^{47}$ and the distribution of dRGC was preferentially located in the temporal and peripheral retinas in mouse ${ }^{47}$ but around the optic disc in monkeys. ${ }^{66}$ The function of dRGC is unknown but was proposed to favor the retinal OFF pathway. ${ }^{47}$ In the present study, dRGCs were detected in the inner margin of the inner nuclear layer, and it is likely that dRGCs were counted in the whole-mounted retinas and were included in the reconstructed retinal maps. Although the fraction of dRGCs was not ascertained in the present study, their presence was sparse and rare. Therefore, it is unlikely that they influenced the pattern of RGC topography due to the low fraction of the dRGC population. 


\subsection{Other cells in the GCL}

In figure 8, several cells in GCL were negative for anti-Brn-3a antibody labeling. Displaced amacrine cells, neuroglial cells and endothelial cells of blood vessels can be found in GCL alongside RGCs. ${ }^{45}$ Traditionally, these cells were identified by their morphological features and by the absence of axons to form the optic nerve during the retrograde labeling. ${ }^{45}$ Endothelial cells of blood vessels in the GCL are relatively easy to identify as they usually have elongated nuclei and a linear arrangement. ${ }^{45}$ Astrocytes and oligodendrocytes are glial cells commonly identified in the retina. ${ }^{45}$ In addition to their small nuclear and cell body size, lack of Nissl substance in the cytoplasm is a main feature of the neuroglial cells. ${ }^{45}$ The problems associated with using morphologic features to differentiate displaced amacrine cells from small RGCs comes from their similar appearances when using cresyl violet staining, especially in regions having a high density of small RGCs. ${ }^{45}$ In one study in which the RGC population of the harlequin tusk fish was determined by both Nissl staining and by retrograde labeling, it was found that more cells were identified as RGCs by cresyl violet staining than by retrograde labeling. ${ }^{48}$ Even so, the topographic distribution of RGCs was not different between two labeling methods. $^{48}$

The ratio of RGCs to non-ganglion cells decreases from center to periphery and may vary among the retinal areas. ${ }^{48}$ In the harlequin tusk fish study, $84-92 \%$ of the cells in the area centrales were labeled with retrograde neural tracer, while only $40-66 \%$ of the cells in the peripheral retinas were identified as RGCs. ${ }^{48}$ In the rabbit study, about $70-80 \%$ of the total retinal neurons were non-ganglion cells in the peripheral retina. ${ }^{46}$ The percentage decreased to $50-60 \%$ in the streak area and $35 \%$ in the areae centrales. ${ }^{46}$ The ratio of RGCs to non-ganglion 
cells in the GCL of the alpaca retinas was not determined in the present study, but it could be estimated if the result of axon count had been valid.

\subsection{Axon counting}

Axon counting from the cross-sectioned optic nerve specimen was included in the present study for the purpose of confirming the accuracy of the RGC labeling by anti-Brn3a antibody. The definition of a RGC is that the axon of the RGC sends the electrical signal received from photoreceptors and interneurons to the retinorecepient zones via the optic nerve. ${ }^{46}$ Thus, in theory, the number of the axons in the optic nerve represent the population of the RGCs in the retina. $^{46}$

The axon count in the present study was 675,188 and the mean (median) population of the positive Brn-3a labeling cells was 416,040 $(408,322)$. The results of axon count and immunohistochemical labeling showed a large discrepancy. It is likely that the discrepancy resulted from the sampling error in the present study. In figure 10 and 11, there was connective tissue located between the optic nerve bundles. When a large amount of connective tissue present in the sampling area, the axon count is relative low. In contrast, when only a small portion of the connective tissue is sampled, the axon count increases significantly. Therefore, the result of the axon count in the sampled area may be significantly influenced by the fraction of the connective tissue. The other possibility of the discrepancy between two results can be only a subpopulation of RGCs was labeled by Brn-3a antibody.

Other limitations of the method in the present study include the presence of unmyelinated axons and the resolution limitation of the light microscope. The presence of the unmyelinated axons was reported in several species, ${ }^{14,16,17}$ and it is like to be present as well in alpacas. Using 
osmium tetroxide successfully identified the axons with myelin in the present study but we were unable to count the unmyelinated axons with this method.

Due to the limitations mentioned above, electron microscopy would be the best method to estimate the axon count in alpacas. It has higher resolution than the light microscope, which allows for the detection of the small axons. Additionally, scanning the specimen with a smaller tile size and increasing the sampling areas are other benefits which could potentially overcome the sampling error.

\subsection{Unsuccessful attempts in Lucifer Yellow retrograde labeling}

Several problems were encountered while conducting the retrograde labeling of LY in this study including the limitations of the dye transportation to the retina and the equipment limitations.

The majority of the published retrograde labeling studies were done on the retinas of small mammals ${ }^{38,47,50}$ and fish. ${ }^{48}$ The large size of the alpaca retina led to a longer distance that the dye needed to travel before reaching the RGC somata. ${ }^{50}$ In addition, the mean (median) of the retinal dimension in alpacas was $891(811) \mathrm{mm}^{2}$ which is much larger than that of the cat or rabbit retina. Because of these size differences, a longer incubation time of LY was attempted to allow the dye to reach the peripheral retinas.

The fact that the eye was no longer living also slowed down the transit time of the dye. ${ }^{50}$ A higher success rate of retrograde labeling with shorter incubation time was reported in vivo study than in ex vivo study due to the active axoplasmic flow in the living eye. ${ }^{38,48,50}$ However, surgeries under general anesthesia are required to perform the retrograde labeling in vivo. ${ }^{38,67}$ This was not possible to perform in the present study. 
The temperature of the incubating medium can also influence the transportation rate of the dye. ${ }^{50}$ It was reported that a longer incubation time was required for the dye to reach the peripheral retina in cool Ames medium versus in the warm medium. ${ }^{50}$ However, in the present study, it was difficult to control the temperature of the medium due to the equipment limitation.

Another equipment limitation encountered in this study included that the argon laser line of the confocal microscope or the filter cubes of the fluorescent microscope only partially covered the excitation and emission wavelengths of LY. This could potentially prevent the detection of LY on the images. An alternative way to detect LY in the retina is to bind LY with anti-LY antibody. With a fluorophore label on anti-LY antibody, the RGC labeled with LY can be detected. Using anti-LY antibody was attempted to overcome this problem in this study. However, lack of control tissue to develop the proper immunohistochemical protocol was another limitation in the study.

Obtaining more healthy alpaca eyes and control tissues with LY would help to optimize the protocols and be the best approach to overcome the limitations mentioned above.

5.8 Unsuccessful attempts to use cresyl violet staining for RGC count

Several different protocols ${ }^{68}$ were used to attempt to obtain distinct staining of the Nissl substance in RGCs, but none was successful. In all the trials, only the nuclei and nucleoli of the cells (not the cytoplasm) were stained. Further research into alternative methods may be necessary to achieve good staining result. 


\section{REFERENCE}

1. Collin S. Behavioural ecology and retinal cell topography In: Archer S, Loew E, Partridge JC, et al., eds. Adaptive Mechanisms in the Ecology of Vision. Great Britain:

Kluwer Academic Publisher, 1999;509-535.

2. Rodieck RW. Visual pathways. Annu Rev Neurosci 1979;2:193-225.

3. Ofri R. Optics and Physiology of Vision In: Gellat KN, Gilger BC,Kern TJ, eds. Veterinary Ophthalmology. USA: Wiley-Blackwell, 2013;208-270.

4. Ofri R. Retina In: Maggs DJ, Miler PE,Ofri R, eds. Slatter's Fundmentals of Veterinary Ophthalmology. St. Louis, Missouri: Saunders, 2008;285-317.

5. Collin SP. A web-based archive for topographic maps of retinal cell distribution in vertebrates. Clin Exp Optom 2008;91:85-95.

6. Salinas-Navarro M, Jimenez-Lopez M, Valiente-Soriano FJ, et al. Retinal ganglion cell population in adult albino and pigmented mice: a computerized analysis of the entire population and its spatial distribution. Vision Res 2009;49:637-647.

7. Samuelson DA. Ophthalmic Anatomy In: Gelatt KN, ed. Veterinary Ophthalmology. 4th ed. Ames, Iowa: Blackwell Publishing, 2007;37-148.

8. Weber AJ, Kaufman PL, Hubbard WC. Morphology of single ganglion cells in the glaucomatous primate retina. Invest Ophthalmol Vis Sci 1998;39:2304-2320.

9. Boycott BB, Wassle H. The morphological types of ganglion cells of the domestic cat's retina. J Physiol 1974;240:397-419.

10. Stone J. A quantitative analysis of the distribution of ganglion cells in the cat's retina. J Comp Neurol 1965;124:337-352. 
11. Stone J, Fukuda Y. Properties of cat retinal ganglion cells: a comparison of Wcells with X-and Y-cells. J Neurophysiol 1974;37:722-748.

12. Rockhill RL, Daly FJ, MacNeil MA, et al. The diversity of ganglion cells in a mammalian retina. $J$ Neurosci 2002;22:3831-3843.

13. Volgyi B, Chheda S, Bloomfield SA. Tracer coupling patterns of the ganglion cell subtypes in the mouse retina. J Comp Neurol 2009;512:664-687.

14. El-Zoghby I, Kassab A. Comparative morphometric study of the optic nerve in one-humped camel (Camelus dromedarius) and donkey (Equus asinus). J Vet Anat 2010;3:23-33.

15. Jonas JB, Schmidt AM, Muller-Bergh JA, et al. Human optic nerve fiber count and optic disc size. Invest Ophthalmol Vis Sci 1992;33:2012-2018.

16. Binggeli RL, Paule WJ. The pigeon retina: quantitative aspects of the optic nerve and ganglion cell layer. J Comp Neurol 1969;137:1-18.

17. Vaney DI, Hughes A. The rabbit optic nerve: fibre diameter spectrum, fibre count, and comparison with a retinal ganglion cell count. J Comp Neurol 1976;170:241-251.

18. Wakakuwa K, Watanabe M, Sugimoto T, et al. An electron microscopic analysis of the optic nerve of the eastern chipmunk (Tamias sibiricus asiaticus): total fiber count and retinotopic organisation. Vision Res 1987;27:1891-1901.

19. Geri GA, Kimsey RA, Dvorak CA. Quantitative electron microscopic analysis of the optic nerve of the turtle, Pseudemys. J Comp Neurol 1982;207:99-103.

20. Krinke A, Frohlich E, Krinke G. An analysis of the distribution of the myelinated nerve fibers in the optic fascicle of a beagle dog. Experientia 1985;41:464-465. 
21. Harman A, Dann J, Ahmat A, et al. The retinal ganglion cell layer and visual acuity of the camel. Brain Behav Evol 2001;58:15-27.

22. Bruesch SR, Arey LB. The number of myelinated and unmyelinated fibers in the optic nerve of vertebrates. J Comp Neurol 1942;77:631-665.

23. Di Scipio F, Raimondo S, Tos P, et al. A simple protocol for paraffinembedded myelin sheath staining with osmium tetroxide for light microscope observation. Microsc Res Tech 2008;71:497-502.

24. Hebel R. Distribution of retinal ganglion cells in five mammalian species (pig, sheep, ox, horse, dog). Anat Embryol (Berl) 1976;150:45-51.

25. Tancred E. The distribution and sizes of ganglion cells in the retinas of five Australian marsupials. J Comp Neurol 1981;196:585-603.

26. Hughes A. The Topography of Vision in Mammals of Contrasting Life Style: Comparative Optics and Retinal Organisation In: Crescitelli F, ed. The Visual system in evolution in vertebrates. Berlin: Springer-Verlag, 1977;615-756.

27. Coimbra JP, Hart NS, Collin SP, et al. Scene from above: retinal ganglion cell topography and spatial resolving power in the giraffe (Giraffa camelopardalis). J Comp Neurol 2013;521:2042-2057.

28. Ofri R. Optics and Physiology of Vision In: Gelatt KN, ed. Veterinary Ophthalmology. 4th ed. Ames, Iowa: Blackwell Publishing, 2007;183-219.

29. Cowey A, Ellis CM. Visual acuity of rhesus and squirrel monkeys. J Comp Physiol Psychol 1967;64:80-84.

30. Reymond L. Spatial visual acuity of the eagle Aquila audax: a behavioural, optical and anatomical investigation. Vision Res. England, 1985;1477-1491. 
31. van Hof MW. Visual acuity in the rabbit. Vision Res 1967;7:749-751.

32. Blake R, Cool SJ, Crawford ML. Visual resolution in the cat. Vision Res $1974 ; 14: 1211-1217$.

33. Jacobson SG, Franklin KB, McDonald WI. Visual acuity of the cat. Vision Res 1976;16:1141-1143.

34. Berkley MA, Watkins DW. Grating resolution and refraction in the cat estimated from evoked cerebral. Vision Res 1973;13:403-415.

35. Harris LR. Contrast sensitivity and acuity of a conscious cat measured by the occipital evoked potential. Vision Res 1978;18:175-178.

36. Harman AM, Moore S, Hoskins R, et al. Horse vision and an explanation for the visual behaviour originally explained by the 'ramp retina'. Equine Vet J 1999;31:384-390.

37. Timney B, Keil K. Visual Acuity in the Horse. Vision Res 1992;32:2289-2293.

38. Pettigrew JD, Dreher B, Hopkins CS, et al. Peak density and distribution of ganglion cells in the retinae of microchiropteran bats: implications for visual acuity. Brain Behav Evol 1988;32:39-56.

39. Bisti S, Maffei L. Behavioural contrast sensitivity of the cat in various visual meridians. J Physiol 1974;241:201-210.

40. Stone $\mathrm{J}$. The number and distribution of ganglion cells in the cat's retina. $J$ Comp Neur 1978;180:753-772.

41. Williams DR, Coletta NJ. Cone spacing and the visual resolution limit. J Opt Soc Am A 1987;4:1514-1523.

42. Maffei L, Fiorentini A. The visual cortex as a spatial frequency analyser. Vision Res 1973;13:1255-1267. 
43. Odom JV, Bromberg NM, Dawson WW. Canine visual acuity: retinal and cortical field potentials evoked by pattern stimulation. Am J Physiol 1983;245:R637-641.

44. Ofri R, Dawson WW, Gelatt KN. Visual resolution in normal and glaucomatous dogs determined by pattern electroretinogram. Prog in Veterinary \& Comparative Ophthalmology 1993;3:111-116.

45. Stone J. The whole mount handbook : a guide to the preparation and analysis of retinal whole mounts: Naitland Publications, 1981.

46. Hughes A. New perspectives in retinal organization In: Osborne NN,Chader G, eds. Progress in Retinal Reseach. New York: Pergamon Press, 1985;243-313.

47. Pang JJ, Wu SM. Morphology and immunoreactivity of retrogradely doublelabeled ganglion cells in the mouse retina. Invest Ophthalmol Vis Sci 2011;52:4886-4896.

48. Collin SP, Pettigrew JD. Retinal ganglion cell topography in teleosts: a comparison between Nissl-stained material and retrograde labelling from the optic nerve. $J$ Comp Neurol 1988;276:412-422.

49. Nadal-Nicolas FM, Jimenez-Lopez M, Sobrado-Calvo P, et al. Brn3a as a marker of retinal ganglion cells: qualitative and quantitative time course studies in naive and optic nerve-injured retinas. Invest Ophthalmol Vis Sci 2009;50:3860-3868.

50. Zhan XJ, Troy JB. An efficient method that reveals both the dendrites and the soma mosaics of retinal ganglion cells. J Neurosci Methods 1997;72:109-116.

51. Lee E-J, Merwine DK, Mann LB, et al. Ganglion cell densities in normal and dark-reared turtle retinas. Brain Research, 2005;40-46.

52. Honig MG, Hume RI. Fluorescent carbocyanine dyes allow living neurons of identified origin to be studied in long-term cultures. J Cell Biol 1986;103:171-187. 
53. Godement $\mathrm{P}$, Vanselow $\mathrm{J}$, Thanos $\mathrm{S}$, et al. A study in developing visual systems with a new method of staining neurones and their processes in fixed tissue. Development 1987;101:697-713.

54. Buchwalow IB, Bocker W. Antibodies for Immunohistochemistry. Immunohistochemistry basics and methods 2010:1-8.

55. Barnstable CJ, Drager UC. Thy-1 antigen: a ganglion cell specific marker in rodent retina. Neuroscience 1984;11:847-855.

56. Fry KR, Tavella D, Su YY, et al. A monoclonal antibody specific for retinal ganglion cells of mammals. Brain Res 1985;338:360-365.

57. Bhide PG, West WC, Fry KR, et al. An immunocytochemical marker for hamster retinal ganglion cells. J Neurocytol 1994;23:167-177.

58. Xiang M, Zhou L, Macke JP, et al. The Brn-3 family of POU-domain factors: primary structure, binding specificity, and expression in subsets of retinal ganglion cells and somatosensory neurons. J Neurosci 1995;15:4762-4785.

59. Dabin I, Barnstable CJ. Rat retinal Muller cells express Thy-1 following neuronal cell death. Glia 1995;14:23-32.

60. Huang W, Fileta J, Guo Y, et al. Downregulation of Thy1 in retinal ganglion cells in experimental glaucoma. Curr Eye Res 2006;31:265-271.

61. Schlamp CL, Johnson EC, Li Y, et al. Changes in Thy1 gene expression associated with damaged retinal ganglion cells. Mol Vis 2001;7:192-201.

62. Provis JM. The Distribution and Size of Ganglion Cells in the Retina of the Pigmented Rabbit: A Quantitative Analysis. J Comp Neurol 1979;185:121-138. 
63. Hoffman E, Fowler ME. The alpaca book. 1st ed. Herald, California: Clay Press, 1995.

64. Dann JF, Buhl EH. Morphology of retinal ganglion cells in the flying fox (Pteropus scapulatus): a lucifer yellow investigation. J Comp Neurol 1990;301:401-416.

65. Bailes HJ, Trezise AE, Collin SP. The number, morphology, and distribution of retinal ganglion cells and optic axons in the Australian lungfish Neoceratodus forsteri (Krefft 1870). Vis Neurosci. England, 2006;257-273.

66. Bunt AH, Minckler DS. Displaced ganglion cells in the retina of the monkey. Invest Ophthalmol Vis Sci 1977;16:95-98.

67. Collin SP, Pettigrew JD. Retinal ganglion cell topography in teleosts: a comparison between Nissl-stained. J Comp Neurol 1988;276:412-422.

68. Ullmann JFP, Moore BA, Temple SE, et al. The Retinal Wholemount Technique: A Window to Understanding the Brain and Behavior. Brain Behav Evol, 2011;1-19. 